AUS $\mid \underset{\text { of Sharjah }}{\substack{\text { Amiversity } \\ \text { She }}}$

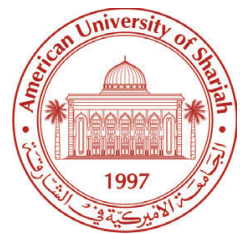

SCHOOL OF BUSINESS ADMINISTRATION WORKING PAPER SERIES

SBAWPS: 01-10/2019

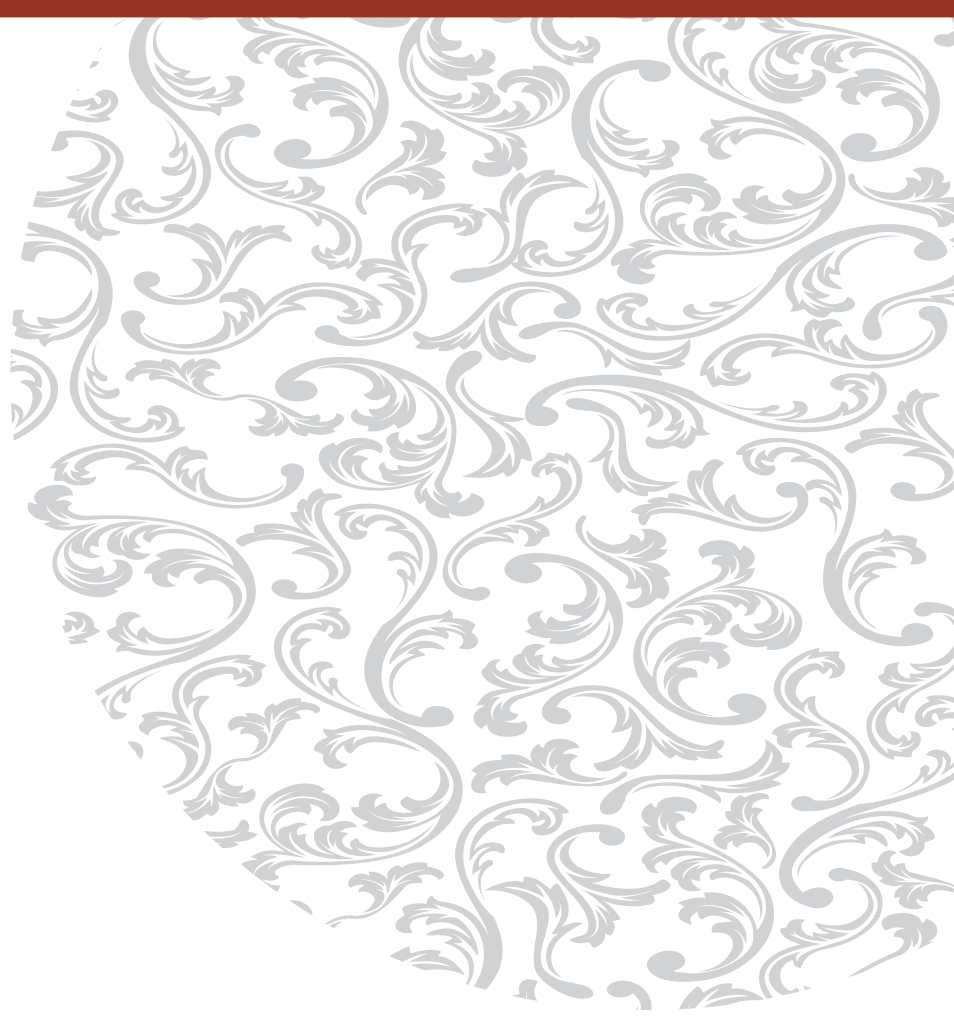

Information Disclosure in Dynamic

Research Contests

Bo Chen

Bo Chen

Dmitriy Knyazev 
Working Paper 01-10/2019

School of Business Administration

Working Paper Series (SBA WPS)

\title{
Information Disclosure in Dynamic Research Contests
}

\author{
Bo Chen, Bo Chen, Dmitriy Knyazev
}

\begin{abstract}
We study the design of information disclosure in a dynamic multi-agent research contest, where each agent privately searches for innovations and submits his best to compete for a winner-takes-all prize. We .nd that although submission is a one-time event for each agent, dixerent disclosure policies on the agents. Submissions induce dixerent equilibrium behavior, making the design of disclosure a useful in-strument for the contest sponsor. We characterize equilibrium behavior in a public contest where submissions are immediately revealed and in a hidden contest where no submission information is revealed to the agents. In addition, for contests with inde.nite duration, the public disclosure policy is an optimal policy among a natural set of disclosure policies.
\end{abstract}

(Keywords: Contest Design, Disclosure Policy, Innovation, Procurement, Research Tournament JEL Classi.cation: C73, D44, D82, D83, O31 ) 


\title{
Information Disclosure in Dynamic Research Contests*
}

\author{
Bo Chen ${ }^{\dagger}$ Bo Chen, ${ }^{\ddagger}$ Dmitriy Knyazev ${ }^{\S}$ \\ September, 2019
}

\begin{abstract}
We study the design of information disclosure in a dynamic multi-agent research contest, where each agent privately searches for innovations and submits his best to compete for a winner-takes-all prize. We find that although submission is a onetime event for each agent, different disclosure policies on the agents' submissions induce different equilibrium behavior, making the design of disclosure a useful instrument for the contest sponsor. We characterize equilibrium behavior in a public contest where submissions are immediately revealed and in a hidden contest where no submission information is revealed to the agents. In addition, for contests with indefinite duration, the public disclosure policy is an optimal policy among a natural set of disclosure policies. (Keywords: Contest Design, Disclosure Policy, Innovation, Procurement, Research Tournament JEL Classification: C73, D44, D82, D83, O31)
\end{abstract}

\section{Introduction}

Research contests, where multiple participants compete to win a prize by searching for a best innovation within a deadline, have been widely regarded as an effective mechanism

${ }^{*}$ We thank Kim-Sau Chung, Daniel Krähmer, Matthias Kräkel, Qingmin Liu, Tymon Tatur, and especially Curt Taylor for helpful discussions, and Jingfeng Lu, Tsz-Ning Wong, Xi Weng, and various seminar and conference participants for constructive comments. The usual disclaimer applies.

${ }^{\dagger}$ Department of Economics, Southern Methodist University, USA (bochen@smu.edu).

${ }_{\ddagger}^{\ddagger}$ Shenzhen Audencia Business School-Shenzhen University, China, and KFUPM Business School, Saudi Arabia (econbc@gmail.com).

${ }^{\S}$ Humboldt University of Berlin, Germany (knyazevd@hu-berlin.de). 
to acquire innovations. There is a long history where various institutions have harnessed research contests to solve complex problems large and small, with one of the earliest being the Longitude Prize offered by the Spanish monarchy during the 1590s. ${ }^{1}$ Research contests were traditionally sponsored by governments and public institutions, due to their influence and financial capability to facilitate participation. However, recent development of information and communication technology has enabled firms, organizations, and even individuals to act as organizers of prized challenges (Piller and Walcher 2006[18]). And internet-based contests have been playing a prominent role for diverse entities to acquire innovative solutions to various tasks, ${ }^{2}$ ranging from mathematical and scientific problems, to complex engineering problems and pressing social and environmental issues. ${ }^{3}$ Recently, the America COMPETES Reauthorization Act passed by Congress in 2010 has also provided Federal agencies with increased investments and authority to conduct prize competitions, reinvigorating the long-standing tradition and importance of research contests in spurring technological innovations for the public.

This paper studies the issue of information disclosure for a dynamic research contest between a sponsor and multiple agents. Previous research on information disclosure in research contests has mainly focused on how to optimally disclose agents' intermediate performance so as to elicit better innovation outcomes. Different from this input-based disclosure in the previous work, we analyze the effects and design of output-based information disclosure policies, i.e., how the sponsor should disclose agents' innovation results so as to achieve favorable outcomes for the sponsor. Our motivation is that in many prized contests, the agents' effort and intermediate research outcomes are typically the agents' private information. Sponsors in these contests only observe the final submitted results over time, and hence only output-based disclosure is feasible. The key insight of the paper is that a strategic disclosure of submitted innovation outcomes, while seemingly indirect and ineffective, can nevertheless alter every agent's incentives throughout the contest, hence also the innovation outcome received by the sponsor.

Our research contest follows and can be regarded as a continuous-time version of that in the seminal paper Taylor (1995)[23], who studies the optimal design of dynamic research

\footnotetext{
${ }^{1}$ The more prominent longitude prize estalished by the British Longitude Act in 1714, which has been cited much more frequently in the innovation literature, actually came after the longitude prizes offered by the Spanish monarchy and the Dutch Republic, but was more successful (Burton and Nicholas 2017[2]).

${ }^{2}$ See www.challenge.gov for challenges offered by the US government, and online innovation platforms such as Kaggle and CrowdAnalytix for contests offered by firms and individuals.

${ }^{3}$ For example, the 2019 MagQuest challenge to measure Earth's magnetic field by the National Geospatial Intelligence Agency ( $\$ 1.2$ million), the 2018 Deloitte XPRIZE for cancer treatment (\$20 million), the 2007-2018 Google Lunar XPRIZE for space innovations (\$30 million), and the 2019 Opioid Detection Challenge by the Department of Homeland Security ( $\$ 1.5$ million).
} 
contests: A sponsor pledges a prize to procure an innovation from several initially identical agents, and the best innovation submitted before a deadline wins the prize. There is no initial uncertainty about the feasibility of the innovation. Each agent searches with cost for innovations, and innovation arrivals for a searching agent follow a Poisson process, with the quality of each innovation modeled as a random draw from a common distribution. At each instant of time, an agent decides whether to search or to stop and submit his best innovation irreversibly. Each agent's past searching history is his private information. The sponsor only observes the submitted innovations from the agents gradually and can decide whether and how to reveal these submissions over time to the (remaining) agents. To focus on information disclosure, we take the number of agents, the prize, and the contest deadline as given and the sponsor's choice only involves publicly committing, before the contest, to a disclosure policy, which delineates how the sponsor plans to disseminate the submission information she receives (if any) over time.

Our analysis starts with two extreme disclosure policies, a hidden policy and a public policy. To put things in perspective, our contest with the hidden policy, the hidden contest, matches the contest studied in Taylor (1995)[23], where the sponsor commits to reveal no submission information throughout the contest and the winner is announced after all agents have submitted their innovations. Our contest with the public policy, the public contest, is one where every submission (its submit time and the submitted innovation) is instantaneously revealed publicly. The public policy was adopted by the prominent 2006 $\$ 1,000,000$ Netflix Grand Prize, which was established to acquire an algorithm that would improve Netflix's old movie recommendation algorithm by $10 \%$. Netflix implemented a real-time leaderboard (a website) where every submission was recorded and 'instantaneously' revealed to the public. ${ }^{4}$

Our first set of results is the equilibrium characterization of the public and hidden contests with a finite horizon. Each contest admits a unique symmetric equilibrium but the agents' behavior differs drastically across the two equilibria. In the hidden contest, an agent adopts a stopping strategy with a constant cutoff. This is similar to that in Taylor (1995)[23]: Receiving no information about the opponents, each agent maintains the same beliefs of the others' progresses and hence employs a constant cutoff in his search. In the public contest, however, absent any submission, each agent plays a stopping strategy with a cutoff that is strictly decreasing over time. This peculiar phenomenon is purely driven by

\footnotetext{
${ }^{4}$ The leaderboard for Netflix Prize can be found at https://www.netflixprize.com/leaderboard.html. As shown on the leaderboard, BellKor's Pragmatic Chaos won the prize in 2009, and BellKor submitted their algorithm only 20 minutes before team The Ensemble. Netlix's contest was extremely successful. However, the winning algorithm was never used, due to practical issues.
} 
information: Though nothing substantial is revealed after a history of no submission, the agents continually receive information about the status of their opponents-for example, they know at least the opponents have not obtained a preemptive innovation. Hence, no information is good information, and accordingly, the agents adjust searching cutoffs downward under such circumstances.

An important implication of our analysis of the public and hidden contests is that agents behave differently under different disclosure policies and hence the design of disclosure policies matters for the sponsor. Our second set of results investigates how the sponsor should strategically design a disclosure policy so as to manipulate the agents' equilibrium behavior to her own benefit. A complete characterization of optimal disclosure policies is however difficult to obtain: Although the sponsor can only disclose submitted innovations, the space of all disclosure policies is still too big to manage. For example, the sponsor can disclose any summary statistic of existing submissions in any dynamic way. In addition, with a finite horizon, a revelation of any submitted innovation alters the behavior of the remaining agents, resulting in ex ante correlation in the agents' equilibrium strategies. In particular, this makes the calculation of the sponsor's expected payoffs under various disclosure policies rather unmanageable.

To make progress, we specialize to infinite-horizon contests. In such contests, deadline effects from a finite horizon, which can permeate and affect behavior throughout the contest, are not present, making equilibrium characterization and analysis of the sponsor's payoffs more viable. We then consider a natural set of $N$ simple disclosure policies where $N$ is the number of agents. In each of the simple disclosure policies, the sponsor announces at most once in the contest, confirming that there have already been $k$ (from 1 to $N$ ) submissions, all passing a cutoff specifically designed for each $k$. These disclosure policies are simple in that they are easy to implement and more importantly they induce simple and unique symmetric equilibria where each agent searches with the designed cutoffs and stops searching once he passes the cutoff or hears the sponsor's announcement. In addition, the hidden and public policies are special 'cases' of the simple disclosure policies in the sense that the simple 1-disclosure (resp., $N$-disclosure) policy induces the same equilibrium outcome as the public policy (resp., the hidden policy).

We show that the simple disclosure policies are strictly ranked from the sponsor's point of view, with simple 1-disclosure policy (the public policy) being the best and simple $N$ disclosure policy (the hidden policy) being the worst. The key intuition behind this ranking result is that different simple disclosure policies expose an agent with different degrees of uncertainty on how likely he is going to win the prize. As a result, the agents 
adopt different search intensity when facing these policies.

The simple disclosure policies, though simple and natural, only constitute a small subset of all possible disclosure policies. However, the ranking result for the simple disclosure policies offers more general insight on how well the sponsor fares under various disclosure policies. The key observation is that ultimately what the sponsor cares about is the equilibrium outcome a disclosure policy induces. We demonstrate that in a sense, the $N$ simple disclosure policies actually generate the entire set of (pure) symmetric equilibrium outcomes in stopping strategies. The public policy (and also the simple 1-disclosure policy) generates the best equilibrium outcome of its kind and is hence an optimal policy among disclosure policies that generate symmetric equilibria in stopping strategies.

While the public policy can be beneficial to the sponsor, information disclosure is a double-edged sword and can also create adversarial incentives for the agents in finitehorizon contests. We demonstrate such caveats in contests with short horizons and with the possibility of strategic delay. In a short-horizon contest, we show via explicit examples that the hidden policy can strictly outperform the public policy. This is again driven by information: In a public contest with a history of no submission, the agents are more and more certain over time that the opponents have not succeeded in good innovations. Immediate revelation of submission then induces the agents to lower equilibrium cutoffs too fast with a short horizon, resulting in less search intensity on average, compared to that in a hidden contest.

Another interesting but adversarial effect of the public policy is that it can induce the agents to strategically delay submissions without searching, particularly in contests where there is no opportunity cost of waiting without submitting - such an assumption is perhaps fitting for online competitions where physical presence in the competition is not required. ${ }^{5}$ The rationale of delay is that in a (finite-horizon) public contest, a submission, unless it is preemptively valued, prompts the remaining agents to search aggressively so as to surpass it. Facing such risk, all agents will then search with a relatively low equilibrium cutoff, sit on their best innovations without submission after passing the cutoff, and wait until a submission from the opponent or the end of the contest. This equilibrium behavior arises as if the agents were coordinating on a low innovation cutoff, albeit in a strictly competitive environment.

\footnotetext{
${ }^{5}$ In our baseline model, an agent incurs a positive flow cost at each instant before submission. The cost can be interpreted as the sum of his opportunity cost and the fiduciary cost from searching. This is a standard assumption. See, e.g., Taylor (1995)[23] and Moscarini and Squintani (2010)[17].
} 


\section{$1.1 \quad$ Related Literature}

Our paper is related to several bodies of work. First, our paper lies squarely in the literature of contests, or all-pay auctions where bids are interpreted as search, effort, or production costs in various competitions. Most of this (extensive) literature however focuses on reduced-form or static contests with complete or incomplete information, and various design issues for such reduced-form contests have been studied (e.g., Fullerton and McAfee 1999[10]; Moldovanu and Sela 2001[16]; Che and Gale 2003[3]). ${ }^{6}$

Our paper is most closely related to the literature on dynamic contests, which is much smaller compared to the literature on static contests. A prominent study in this literature is Taylor (1995)[23], who analyzes a fixed-prize dynamic contest among symmetric agents, and the innovation process is modeled as an optimal stopping problem with costly search and random innovations. Taylor (1995)[23] characterizes the optimal contest design using instruments such as the number of participants, entry fee and contest prize. Our model, as mentioned, extends and builds on Taylor's model, but our paper differs in that we focus on the design of (submission) disclosure policies, taking other design instruments as fixed. In particular, the contest in Taylor (1995)[23] is a (discrete-time) hidden contest in our setting and our analysis implies that information disclosure can be yet another effective and useful instrument for the sponsor to improve the design of the contest, particularly so if the contest horizon is sufficiently long. ${ }^{7}$

There has also been a recent literature that studies dynamic contests in continuous time (Seel and Strack 2013[20]; Lang, Seel and Strack 2014[15]; Seel and Strack 2016[21]). While we also consider continuous-time contests with a winner-takes-all payoff structure and privately observed stochastic research progresses, and our equilibrium existence and characterization share some technical similarities with those in this literature, our contest game is relatively simple and our objective is to study information disclosure, which is absent in these studies. In a similar vein, our paper is also related to the literature on innovation contests with learning, in particular to Halac, Kartik and Liu (2017)[13]. ${ }^{8}$ Halac, Kartik and Liu (2017)[13] study optimal contest design using instruments such as disclosure policies, prize-sharing schemes and prizes in a contest with initial uncertainty on innovation feasibility. Our paper differs from Halac, Kartik and Liu (2017) in that

\footnotetext{
${ }^{6}$ The literature on static contests and all-pay auctions is large and ongoing. A review of this literature can be found in several recent and excellent surveys (e.g., Corchón (2007)[5] and Konrad (2009)[14]).

${ }^{7}$ Our result that a public contest is strictly better than a hidden contest is established in a setting with indefinite duration. However, the same result holds for contests with finite but sufficiently long horizons (the proof is available upon request). This can also be seen in our numerical analysis in Figure 1.

${ }^{8}$ For brevity, we refer to Halac, Kartik and Liu (2007)[13] for an illustration of this extensive literature.
} 
we only focus on the design of disclosure policies, and our setting is different in that the set of each agent's states here is a continuum, rather than binary. In particular, the difference in our setting has substantial implications on agents' search behavior, the sponsor's incentives, and the set of disclosure policies available to the sponsor. ${ }^{9}$ As such, the two settings are not directly comparable and our results are not subsumed by those in Halac, Kartik and Liu (2017)[13].

On the issue of information disclosure, our paper relates to the literature on how a principal should disclose agents' intermediate performance in multistage contests (e.g., Yildirim 2005[24]; Gill 2008[11]; Zhang and Wang 2009[25]; Aoyagi 2010[1]; Ederer 2010[7]; Rieck 2010[19]; Goltsman and Mukherjee 2011[12], and Daley and Wang 2018[6]). Overall, these papers have studied various issues such as timing of disclosure ([6]), desirability of interim feedback ([1], [7], [19]), optimal disclosure ([12], [25]), and voluntary disclosure ([24], [11]) in different settings of dynamic contests. Different from these studies, the sponsor in our setting only observes (and discloses) information about the agents' final outputs. As such, the information requirement for our sponsor is different and our disclosure has different effects on the agents' incentives. Nevertheless, our paper complements these prior studies in showing that strategic disclosure of the agents' final outputs can also be effective in a dynamic contest.

More broadly, our paper is related the growing literature on designing information feedback in dynamic settings (e.g., Ely 2017[8], Che and Hörner 2018[4], Ely and Szydlowski 2017[9], etc.) where a designer chooses how to optimally disseminate valuable information over time as the only incentive instrument to motivate agents. Compared to this literature, our framework is unique in that there are multiple forward-looking agents and the information to be disclosed is generated dynamically and endogenously in our contest. Moreover, our results are consistent with this literature in showing that information design can be an effective instrument in incentivizing agents in dynamic settings.

\section{The Innovation Model}

A sponsor or principal (she) organizes a dynamic research contest with a fixed deadline $T \leq \infty$ and offers a pre-specified prize, normalized to 1 , to acquire an innovation. There are $N \geq 2$ risk-neutral participating agents (he) who can search for innovations before

\footnotetext{
${ }^{9}$ To illustrate, a 'successful' innovation in our setting is now endogenously determined in equilibrium, and so is the value of a successful innovation to the sponsor. In addition, a disclosure policy in our setting can specify not only who has submitted but also what the submitted value is.
} 
$T$. We take the prize and the deadline as exogenously given so as to focus on the role of information disclosure.

Searching for innovations requires resources from each agent, which we model as a flow of search cost of $c>0$ per unit time. We interpret $c$ as the fiduciary and opportunity cost of (per unit of) time for conducting research. ${ }^{10}$ The arrival of innovations for an agent while searching follows a time-homogeneous Poisson process with a (publicly known) arrival rate $\lambda$. The value or the state of each arrived innovation, denoted as $a$, is drawn independently across time and among agents from a common differentiable distribution function $F$ on $[0,1]$ with a positive, bounded density function $f$. We assume that whether an agent is actively searching and the values of his arrived innovations are the agent's private information. To avoid less interesting cases, we assume that the search cost $c$ is sufficiently small so that $c<\frac{\lambda}{N} \cdot{ }^{11}$

All agents start from an initial state of $a=0$, which is assumed to be worthless and unable to win the contest. Hence an agent has to search and obtain a positive innovation so as to (possibly) win the prize. At each instant, each agent makes a decision on whether to search or stop. If an agent stops at instant $t$, he decides whether to submit his best innovation up to time $t$ (i.e., each agent searches with perfect recall) and exits irreversibly from the contest. The prize is awarded to the agent with the highest submitted innovation value, with ties broken randomly. We assume that the value of each submitted innovation can (only) be verified at no cost by the sponsor, and that the scrap value of an innovation not winning the prize is zero to the agents and the sponsor.

We now describe the sponsor's objective, information, and strategies. The sponsor only needs one innovation and an innovation with state $a$ is worth $v(a)$ to the sponsor, and $v(a)$ is strictly increasing in $a$ (hence the sponsor can be risk-averse or risk-loving). ${ }^{12}$ Given that search behavior and search outcomes are the agents' private information and submitted innovations have to be verified by the sponsor, we take the realistic position that the sponsor only observes the identities, timing, and values of all submissions over time. Formally, denote the submission status of $i$ at time $t$ as $s_{i, t} \in\left\{\varnothing,\left(\tau_{i}, \omega_{i}\right)\right\}$ where $s_{i, t}=\varnothing$ if agent $i$ has not submitted at time $t$ and $s_{i, t}=\left(\tau_{i}, \omega_{i}\right)$ if agent $i$ made a submission at time $\tau_{i} \leq t$ with submitted state $\omega_{i} \in[0,1]{ }^{13}$ The sponsor's information

\footnotetext{
${ }^{10}$ This interpretation of $c$ is similar to that in Taylor (1995)[23]. We differentiate search cost from opportunity cost of time and analyze the corresponding equilibrium in Section 5 .

${ }^{11}$ If $c>\lambda$, no agent has incentive to search at any time for innovations. The restriction of $c<\frac{\lambda}{N}$ rules out situations where only some (but not all) agents participate in the contest in equilibrium.

${ }^{12}$ One might also want to impose $v(a)>1$ for all $a>0$ - together with $c<\lambda / N$, this implies that active search by at least some agents is efficient.

${ }^{13}$ Given irreversible exit, an agent can only make at most one submission. Hence, if an agent makes a
} 
at $t$ is a history $\left(\mathbf{s}_{t}\right)_{\tau \leq t}$ where $\mathbf{s}_{t}=\left(s_{1, t}, \ldots, s_{n, t}\right)$ is the contest submission status at $t$. Before the contest begins, the sponsor announces and commits publicly to a disclosure policy D. For finite-horizon contests (in Section 3), two extreme information disclosure policies will be especially important, a public policy where the sponsor fully discloses $\mathbf{s}_{t}$ in each instant $t$, and $a$ hidden policy where the sponsor does not reveal any information about $\mathbf{s}_{t}$ throughout the contest. The public policy can be implemented by, for example, establishing a leaderboard showing the submission status $\mathbf{s}_{t}$ in real time. We will consider and analyze general disclosure policies in Section 4.

Each agent's (behavior) strategy is to decide whether to search and if not, whether to submit at each instant $t$, given his realized innovations up to time $t$ and possibly revealed information about the opponents' submissions. Each agent's objective is to maximize his probability of winning the prize, net of his flow of search cost. To simplify notation, we assume no discounting. Finally, we use the solution concept of Nash equilibrium for the contest game under various disclosure policies and we restrict attention to symmetric equilibrium in which all agents use the same strategy. ${ }^{14}$

It is well known that there are various technical difficulties in modeling games in continuous time, in that basic discrete-time concepts such as decision trees and strategies have no direct continuous-time analogs (Simon and Stinchcombe 1989[22]). ${ }^{15}$ A related problematic issue arises in our contest when an agent is called upon to respond to some disclosed information in the middle of the contest. To address this, we adopt the specification that once some information (e.g., a submission or a summarizing statistic for $\mathbf{s}_{t}$ at $t$ ) is publicly disclosed, a buzzing notification sound (say, on the real-time leaderboard) is played, effectively stopping all remaining agents from searching for an (arbitrarily) small duration of time. The remaining agents then make further decisions after the notification. As long as such notifications only occur finitely many times in the game, such a specification does not qualitatively change our results.

Finally, the dynamic contest game unfolds according to the following time line: First, the sponsor publicly commits to an information disclosure policy. The agents then compete by searching for innovations. Finally, upon receiving a submission, the sponsor discloses (certain) information about the submission according to her announced disclosure policy. After receiving all submissions, the sponsor announces the best innovation

submission with $\omega_{i}$ at $\tau_{i}$, then $s_{i, t}=\left(\tau_{i}, \omega_{i}\right)$ for all $t \geq \tau_{i}$.

${ }^{14}$ We will nevertheless describe off-the-equilibrium-path behavior as well in our characterization of the symmetric Nash equilibrium of the public contest game.

${ }^{15}$ For example, a simple trigger strategy in discrete time can be difficult to formulate in a continuoustime game (as the real numbers are not well-ordered). 
and the corresponding winning agent, and awards the prize to the winning agent.

Remark 1 Given our assumption that the search cost $c$ is an agent's cost of staying in the contest in each instant, an agent immediately submits his best innovation once he stops searching for innovations, since waiting incurs the same cost without generating any innovation. While this assumption is not uncommon and can be reasonable in those contests that require physical presence, ${ }^{16}$ it can be too strong in some other contests (e.g., online contests hosted on Kaggle). We relax this assumption in Section 5, where we differentiate fiduciary search cost from opportunity cost of time, and analyze equilibrium outcomes where agents strategically wait and submit their innovations at a later time.

\section{Equilibrium Analysis}

We now characterize the symmetric equilibrium of the dynamic contest under the public and the hidden information disclosure policies. We call the contest under the public (resp., hidden) policy a public contest (resp., a hidden contest). We start our analysis with twoagent hidden and public contests, which admit explicit equilibrium characterization, and facilitate a more intuitive and clear discussion of equilibrium outcomes. The analysis of the two-agent contests also provides useful foundation for equilibrium characterization of the general $N$-agent contests established later in this section.

\subsection{The Two-Agent Hidden Contest}

Consider the hidden contest where the sponsor conceals submission information until receiving both submissions. The hidden contest here is qualitatively identical to the (discrete-time) setting in Taylor (1995)[23], except that ours is in continuous time with Poisson innovation arrivals. In the game, each agent's strategy is a sequence of statedependent decisions on whether to stop searching and if yes, whether to submit his innovation. As in Taylor (1995)[23] and the job search literature, it is a standard result that an agent's optimal strategy is a stopping strategy, i.e., to continue searching until the agent reaches above a constant state of innovation, at which point, the agent makes a submission. Each agent only observes his private history, which consists of his previous innovation arrival times and his innovation quality for each arrival. We characterize the (unique) symmetric Nash equilibrium in cutoff strategies.

\footnotetext{
${ }^{16} \mathrm{~A}$ similar assumption appears for example in the experimentation model with incomplete information in Moscarini and Squintani (2010)[17].
} 
First, denote $Z\left(a \mid a^{\prime}, T\right)$ as the time-0 cumulative distribution function for an agent's ultimate best innovation at $T$ to be below $a \in[0,1]$ when the agent uses an $a^{\prime}$-cutoff strategy. We have

$$
Z\left(a \mid a^{\prime}, T\right)=\left\{\begin{array}{l}
0, \text { if } a=0 \\
e^{-\lambda T[1-F(a)]}, \text { if } a \in\left(0, a^{\prime}\right] \\
e^{-\lambda T\left[1-F\left(a^{\prime}\right)\right]}+\left[1-e^{-\lambda T\left[1-F\left(a^{\prime}\right)\right]}\right] \frac{F(a)-F\left(a^{\prime}\right)}{1-F\left(a^{\prime}\right)}, \text { if } a \in\left(a^{\prime}, 1\right]
\end{array} .\right.
$$

Notice that in (1), given the $a^{\prime}$-cutoff strategy, the term $e^{-\lambda T[1-F(a)]}$ is the probability that the agent's best draw is below $a \leq a^{\prime}$, while $\frac{F(a)-F\left(a^{\prime}\right)}{1-F\left(a^{\prime}\right)}$ is the conditional probability that the innovation is above the cutoff $a^{\prime}$ and below $a$.

Next, suppose agent $i$ 's current state is $a \geq 0$ and agent $j$ uses an $a^{*}$-cutoff strategy. Agent $i$ 's instantaneous gain from searching at state $a$ is

$$
\Delta \Pi\left(a, a^{*}\right)=\lambda \int_{a}^{1}\left[Z\left(x \mid a^{*}, T\right)-Z\left(a \mid a^{*}, T\right)\right] d F(x)-c .
$$

The term $\Delta \Pi\left(a, a^{*}\right)$ describes agent $i$ 's marginal incentives to search at state $a$ : Facing opponent $j$ with an $a^{*}$-cutoff strategy, $i$ 's expected payoff from stopping at state $a$ is exactly $Z\left(a \mid a^{*}, T\right)$ — recall that agent $i$ does not observe $j$ 's state and hence $i$ 's belief, or the distribution of $j$ 's state at $T$, stays the same throughout the game, regardless of $i$ 's private history. Searching in the next instant and then stopping results in either a state below $a$, leading to the same payoff $Z\left(a \mid a^{*}, T\right)$, or a state above $a$, with a payoff of $\int_{a}^{1} Z\left(x \mid a^{*}, T\right) d F(x)$. Hence, $\Delta \Pi\left(a, a^{*}\right)$ is $i$ 's additional expected payoff net of cost $c$.

Proposition 1 characterizes the unique symmetric equilibrium of the hidden contest:

Proposition 1 (Hidden Contest with Two Agents) The hidden contest game with $T<\infty$ admits a unique symmetric equilibrium, in which both agents search with a timeindependent cut-off $a^{*}$ :

1. If $\frac{\lambda\left(1-e^{-\lambda T}\right)}{2} \leq c<\frac{\lambda}{2}$, both agents search with cutoff $a^{*}=0$;

2. If $0<c<\frac{\lambda\left(1-e^{-\lambda T}\right)}{2}$, both agents search with an interior cutoff $a^{*} \in(0,1)$ such that

$$
\lambda\left[1-F\left(a^{*}\right)\right]\left[1-e^{-\lambda T\left(1-F\left(a^{*}\right)\right)}\right]=2 c .
$$

Proposition 1 is intuitive: When search cost is relatively large (Case 1), each agent will stop searching once he successfully obtains a single draw from $F$, regardless of its 
realization. In other words, both agents search with the minimal cutoff value $a^{*}=0$, which can be regarded as a corner solution. The agents will search more intensively in equilibrium (i.e., with an interior cutoff $a^{*}$ ) when search cost is small (Case 2). To view the parameter restrictions in Proposition 1 from a different angle, fixing $c$ and $\lambda$, Case 1 indicates that if the horizon of the contest is relatively short, i.e., $T \leq T^{*}=-\frac{\ln (1-2 c / \lambda)}{\lambda}$, then both search with the minimal cutoff $a^{*}=0$, and the agents search more intensively when the horizon is bigger than $T^{*}$.

Importantly, each agent in the hidden contest receive no information about the opponent and hence adopts a stationary stopping value $a^{*}$ that is invariant in time throughout the contest. As we will see, this stands in sharp contrast with the agents' equilibrium behavior in the public contest.

\subsection{The Two-Agent Public Contest}

In the public contest, a submission is immediately revealed to the public. An agent's strategy here hence should also specify his behavior after a submission from the opponent. We now characterize the symmetric equilibrium in cutoff strategies of the public contest where the cutoff now depends on both the calendar time and the opponent's submission.

First, consider a history where $j$ submits a lucky draw with a realized value $a_{j} \geq \hat{a}=$ $F^{-1}\left(1-\frac{c}{\lambda}\right)$. Agent $i$ 's instantaneous gain from searching given submission $a_{j}$ is

$$
\lambda\left[1-F\left(a_{j}\right)\right]-c \leq \lambda[1-F(\hat{a})]-c=0 .
$$

The innovation $\hat{a}$ is thus a threshold, which once submitted will forestall further search from the opponent, hence guaranteeing winning for an agent. Given this, an agent with an innovation at least $\hat{a}$ after a history of no submission stops searching and submits his innovation immediately in equilibrium.

The above analysis also implies that after a history where $j$ has submitted an innovation $a_{j}<\hat{a}$, agent $i$ should continue searching, but should do so with cutoff $a_{j}$, i.e., agent $i$ stops searching and submits his best innovation whenever it is above $a_{j}$-additional search only incurs wasteful cost without any additional benefits.

Next, consider histories where no one has yet made a submission. While an innovation of value $\hat{a}$ makes the opponent idle and effectively wins the contest, "searching with cutoff value $\hat{a}$ when there is no previous submission" however cannot be part of an equilibrium strategy when $T$ is finite. To see that, consider a history with no submission and at a time close to the deadline $T$. If agent $i$ 's best innovation is less than but close to $\hat{a}$, it 
is then optimal for agent $i$ to stop searching and submit his best innovation, since the additional expected benefit from searching is too small to justify the search cost given the approaching deadline. ${ }^{17}$ Things then unravel in that each agent then searches with even lower cutoffs than $\hat{a}$ at earlier time points, and so on. We will see, however, that such an end-point effect is not present in an infinite-horizon public contest, in which each agent indeed searches with cutoff $\hat{a}$ after a history of no submission.

Now define an agent's expected payoff from stopping (and submitting) at state $a_{t} \in$ $[0, \hat{a})$ at instant $t<T$ as $V\left(a_{t}\right)$ : Here $a_{t}$ is the best innovation drawn up to calendar time $t$ and we suppress subscript $i$ due to symmetry. We have

$$
V\left(a_{t}\right)=\left\{\begin{array}{l}
1, \text { if } a_{t} \geq \hat{a} \\
\mu_{t} e^{-\lambda(T-t)\left[1-F\left(a_{t}\right)\right]}, \text { if } a_{t} \in(0, \hat{a}) \\
0, \text { if } a_{t}=0
\end{array}\right.
$$

where $\mu_{t}=\operatorname{Pr}\left(a_{j}<a_{t} \mid H_{t}\right)$ is an agent's belief that his opponent's current state is below $a_{t}$ given the agent's (private) history at $t$, denoted as $H_{t}$, which consists of the agent's past Poisson arrivals with the associated realized innovations before time $t .{ }^{18}$ Equation (3) is intuitive: An innovation with $a_{t}=0$ cannot win the contest. In addition, when an agent submits $a_{t} \in\left[0, \hat{a}\right.$ ), the opponent (with a state below $a_{t}$ ) will search with cutoff $a_{t}$ until $T$. The agent will then only receive the prize (of 1 ) when the opponent fails to find an innovation above $a_{t}$ after $t$, which occurs with probability $e^{-\lambda(T-t)\left[1-F\left(a_{t}\right)\right]}$.

Denote the instantaneous gain from searching at state $a_{t} \in(0, \hat{a})$ and instant $t$ (and then stopping) after a history of no submission as $\Delta \Pi\left(a_{t}\right)$ :

$$
\Delta \Pi\left(a_{t}\right)=\lambda \int_{a_{t}}^{\hat{a}} V(a) d F(a)-\lambda\left[1-F\left(a_{t}\right)\right] V\left(a_{t}\right) .
$$

In deriving $\Delta \Pi\left(a_{t}\right)$, notice that (i) a (successful) instantaneous search can either draw an innovation below $a_{t}$ resulting in the same payoff $V\left(a_{t}\right)$, or a state above $\hat{a}=F^{-1}\left(1-\frac{c}{\lambda}\right)$ leading to a submission, or a state in between $a_{t}$ and $\hat{a}$, and (ii) the probability that the opponent succeeds and submits his innovation can be ignored. As before, $\Delta \Pi\left(a_{t}\right)$ describes an agent's marginal incentives to search at $a_{t}$, i.e., an agent searches for the

\footnotetext{
${ }^{17}$ Indeed, an agent will have no incentive to search at states near $\hat{a}$ and this is true regardless of $t$. A more rigorous demonstration of this intuition can be found in the proof of Proposition 2 .

${ }^{18}$ The belief $\mu_{t}$ can be analytically calculated given that the agents' states are independent. Specifically, in the symmetric equilibrium (to be constructed), $\mu_{t}=\mu_{t}\left(a_{t}\right)=e^{-\lambda t\left[1-F\left(a_{t}\right)\right]}>e^{-\lambda t}>0$ where $e^{-\lambda t}$ is the probability that the opponent receives no innovation opportunities before $t$. As we will see, however, the belief $\mu_{t}$ is irrelevant for an agent's stopping decision as long as $\mu_{t}>0$.
} 
next instant if $\Delta \Pi\left(a_{t}\right)>0$ and stops if $\Delta \Pi\left(a_{t}\right)<0$. Importantly, observe that while an agent's belief $\mu_{t}$ in (3) affects the value $V\left(a_{t}\right)$, this belief does not depend on $a_{t} \in(0, \hat{a})$ and hence does not affect an agent's stopping decision, as long as $\mu_{t}>0$.

Let $\alpha_{t}^{*}$ be the value at which an agent is indifferent between stopping and searching for the next instant, i.e., $\Delta \Pi\left(\alpha_{t}^{*}\right)=0$. And $\alpha_{t}^{*}$ is implicitly determined by

$$
\lambda \int_{\alpha_{t}^{*}}^{\hat{a}} V(a) d F(a)=\lambda\left[1-F\left(\alpha_{t}^{*}\right)\right] V\left(\alpha_{t}^{*}\right) .
$$

It can be shown that $\Delta \Pi\left(a_{t}\right)$ is strictly decreasing in $a_{t}$, and if a solution $\alpha_{t}^{*} \in[0, \hat{a}]$ for $(4)$ exists, it will be unique. In addition, as in the hidden contest, the indifference condition (4) may not admit a solution in $[0, \hat{a}]$ and we can have a corner solution $\left(\alpha_{t}^{*}=0\right)$. However, different from the hidden contest, we can now have a corner solution for $\alpha_{t}^{*}$ either at the beginning of the game (i.e., when $T$ is small) or at some $t$ close to the deadline $T$. To see this, consider the scenario where $a_{t}=0$ at some $t$, i.e., an innovation opportunity has not arrived up to $t$. At this point, while the agent always has strict incentives to search at state $a_{t}=0$, there may not exist an interior $\alpha_{t}^{*} \in(0, \hat{a})$ solving the indifference condition (4), particularly so if $t$ is sufficiently close to $T$. The intuition is that an agent in such a situation believes that most likely his opponent has not received any innovation opportunity (just like himself). Given the approaching deadline, the agent then submits immediately once he gets an innovation above 0 .

Proposition 2 characterizes the unique symmetric equilibrium of the public contest:

Proposition 2 (Public Contest with Two Agents) The public contest game with $T<$ $\infty$ admits a unique symmetric equilibrium:

1. When there is no previous submission, both agents search at $t<T$ with a unique time-dependent cutoff value $\alpha_{t}^{*} \in[0, \hat{a})$ where $\alpha_{t}^{*}$ is implicitly defined in (4) whenever a solution exists, and $\alpha_{t}^{*}=0$ otherwise. An agent searches whenever $a_{t}$, his best innovation up to time $t$, is such that $a_{t} \leq \alpha_{t}^{*}$, and the agent submits $a_{t}$ otherwise.

2. When the opponent $j$ submits an innovation with value $a_{j}<\hat{a}=F^{-1}\left(1-\frac{c}{\lambda}\right)$, agent $i$ continues to search with $a_{j}$ as the cutoff value until $T$, and agent $i$ stops searching immediately (submits his best innovation and loses) if $a_{j} \geq \hat{a}$.

Hence, in the public contest, the agents start their search with a time-dependent cutoff $\alpha_{t}^{*}$. An agent immediately submits his best innovation $a_{t}$ whenever $a_{t}>\alpha_{t}^{*}$. Seeing a 
submission with innovation $a_{t}$, the opponent either continues to search with the submitted $a_{t}$ as the cutoff (if $a_{t}<\hat{a}$ ) or stops searching (if $a_{t} \geq \hat{a}$ ).

Simplifying (4) using (3), the cutoff $\alpha_{t}^{*}$ is implicitly defined by:

$$
\frac{e^{\left[\lambda\left(1-F\left(\alpha_{t}^{*}\right)\right)-c\right](T-t)}-1}{T-t}=\lambda\left[1-F\left(\alpha_{t}^{*}\right)\right] .
$$

Observe that as $t \uparrow T$, the LHS of (5) converges to " $\lambda\left[1-F\left(\alpha_{t}^{*}\right)\right]-c$ ", which is strictly less than the RHS of (5). Hence, (5) admits no interior solution for $\alpha_{t}^{*}$ at times close to $T$. As noted above, we impose $\alpha_{t}^{*}=0$ in this scenario, which more explicitly arises if $e^{(\lambda-c)(T-t)}-1 \leq \lambda(T-t)$. Moreover, it can be verified (via implicit differentiation) that $\alpha_{t}^{*}$ strictly decreases in $t$ for interior $\alpha_{t}^{*}$, the initial cutoff $\alpha_{0}^{*}$ strictly increases in $T$, and $\alpha_{0}^{*}$ approaches $\hat{a}$ as the deadline $T$ approaches $\infty$, i.e.,

$$
\frac{d \alpha_{t}^{*}}{d t}<0, \frac{d \alpha_{0}^{*}}{d T}>0, \text { and } \lim _{T \rightarrow \infty} \alpha_{0}^{*}=\hat{a}=F^{-1}\left(1-\frac{c}{\lambda}\right)
$$

To compare the agents' equilibrium behavior in the hidden and public contests, consider the following simulation results (Figure 1) on the cutoffs $a^{*}$ in Proposition 1 and $\alpha_{t}^{*}$ (as a function of $t$ ) in Proposition 2:
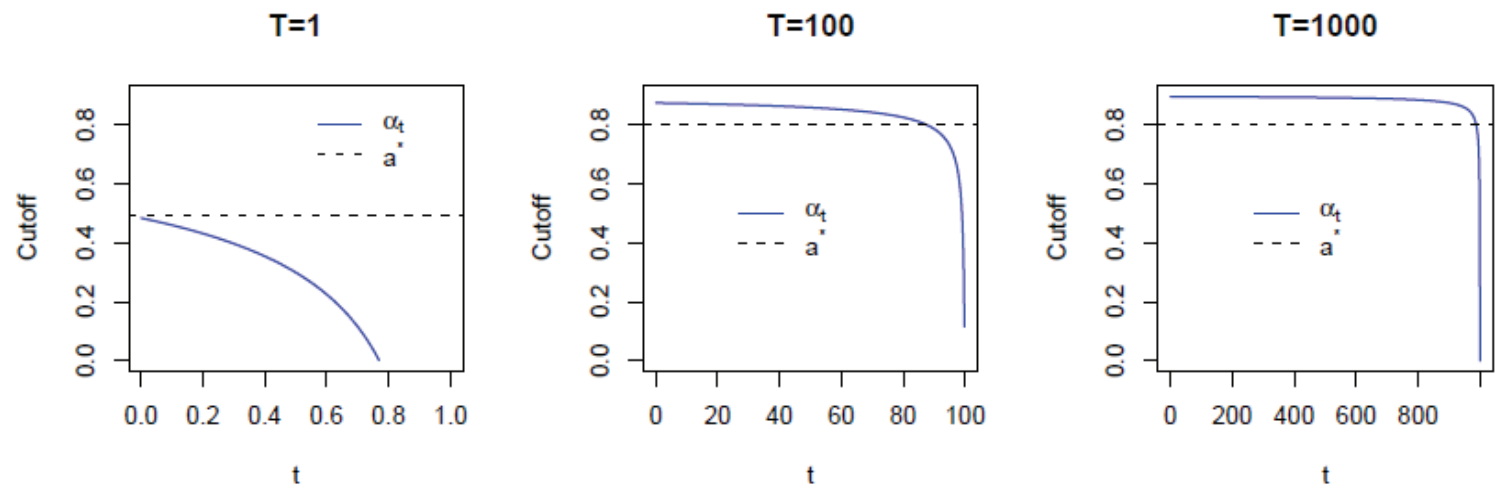

Figure 1. Equilibrium Cutoff Values of the Public and Hidden Contest.

Cutoff $a^{*}$ : dotted horizontal line; Cutoff $\alpha_{t}^{*}$ : solid blue curve $(\lambda=1, c=0.1, F$ : Uniform).

Figure 1 shows that with a sufficiently long horizon $(T=100$ and $T=1000)$, the agents start searching with a higher cutoff $\left(\alpha_{0}^{*}>a^{*}\right)$, and hence search more intensively, in a public contest, than in the hidden contest. However, when approaching the end of the contest, if there is still no submission from anyone, the looming deadline prompts each agent to adopt a cutoff rule that drops quickly toward zero. When the contest deadline is short $(T=1)$, however, we have $\alpha_{t} \leq a^{*}$ throughout the contest, implying that ex ante 
both agents can search less intensively in the public contest than in the hidden contest, which can make the hidden contest strictly better for the sponsor. ${ }^{19}$

It is perhaps surprising that a seemingly minor change in the disclosure policy would lead to drastically different searching behavior from the agents. As mentioned before, in the hidden contest, given the same belief of the opponent's progress throughout the game, each agent adopts a time-independent cutoff in equilibrium. One particular uncertainty preventing each agent from searching more intensively is the possibility that the opponent has already obtained a very good innovation, in which case a continual search for an agent is a pure waste of resources.

In the public contest, although a submission from an agent is a one-time event, each agent actually receives information continually about the opponent's progress: When there is no submission, each agent knows that no one has achieved a "preemptive" innovation and each agent hence always has incentives to continue to search. On the other hand, when the opponent submits his innovation, there is then no uncertainty about the opponent's state, and continual searching using the opponent's submitted innovation as a cutoff can never be futile (in expectation). A similar rationale explains why an agent's equilibrium cutoff drops quickly after a history of no submission when the deadline is approaching: At such times, each agent is more and more certain that the opponent either has not received any innovation arrival or has not obtained a good innovation. It then makes little sense for him to search aggressively and each agent hence optimally responds by using a smaller cutoff value with the approaching deadline.

Overall, the difference in the equilibrium behavior of the hidden and public contests is purely driven by informational considerations. Compared to the public contest, the agents in the hidden contest do not know each other's progress throughout the game. Such lack of information leaves the agents completely uncertain of the opponent's state, damping both agents' incentives to search, especially in a sufficiently long contest.

\subsection{General $N$-Agent Contests}

We now analyze symmetric equilibria for the $N$-agent contest games. Naturally, this general setting complicates the analysis, especially for the $N$-agent public contest. As a result, the equilibrium outcomes are more obscured. Nevertheless, we are able to characterize

\footnotetext{
${ }^{19}$ This statement is obtained only from eyeballing Figure 1 without rigorous justification. A precise calculation on this is difficult, given that ex ante, the agents' search behavior is correlated in the public contest (for example, it is possible for an agent to submit $a \in\left(a^{*}, \hat{a}\right)$ in the public contest and the other agent then searches with $a$ as the cutoff, which can result in an innovation even bigger than $\left.a^{*}\right)$. We will come back to this issue and compare the two contests with short horizons more rigorously in Section 5 .
} 
the structure and uniqueness of symmetric equilibrium in this general setting.

\subsubsection{The Hidden Contest}

First, recall $Z\left(a \mid a^{*}, T\right)$ in (1), which is the time-0 cumulative distribution function of an agent's best innovation at $T$ below $a \in[0,1]$ with an $a^{*}$-cutoff strategy. Agent $i$ 's instantaneous gain from searching at state $a$ is: ${ }^{20}$

$$
\Delta \Pi\left(a, a^{*}\right)=\lambda \int_{a}^{1}\left[Z^{N-1}\left(x \mid a^{*}, T\right)-Z^{N-1}\left(a \mid a^{*}, T\right)\right] d F(x)-c .
$$

As in $(2), \Delta \Pi\left(a, a^{*}\right)$ is the additional expected payoff net of cost $c$ from searching in the next instant.

Define the equilibrium cutoff $a^{*} \in[0,1]$ as $\Delta \Pi\left(a^{*}, a^{*}\right)=0$, or equivalently

$$
\lambda \int_{a^{*}}^{1}\left[Z^{N-1}\left(x \mid a^{*}, T\right)-Z^{N-1}\left(a^{*} \mid a^{*}, T\right)\right] d F(x)=c .
$$

The existence and uniqueness of a symmetric equilibrium in the hidden contest game are then implied by each agent's marginal incentives to search at each instant $t$ and state $a$ in (7), and are equivalent to the existence and uniqueness of a solution to (8).

Define a cost threshold $c^{*}$ as

$$
c^{*}=\frac{\lambda}{N} \sum_{k=0}^{N-1}\left(e^{-k \lambda T}-e^{-(N-1) \lambda T}\right) .
$$

And $c^{*}$ reduces to the threshold $\lambda\left(1-e^{-\lambda T}\right) / 2$ in Proposition 1 when $N=2$. Proposition 3 characterizes the unique symmetric equilibrium of the $N$-agent hidden contest: ${ }^{21}$

Proposition 3 (Hidden Contest with $N$ Agents) The $N$-agent hidden contest with deadline $T<\infty$ admits a unique symmetric equilibrium, in which all agents search with an identical and time-independent cut-off $a^{*}$ :

1. If $c \in\left[c^{*}, \frac{\lambda}{N}\right)$, all agents search with cutoff $a^{*}=0$;

\footnotetext{
${ }^{20}$ We write $\left[Z\left(a \mid a^{*}, T\right)\right]^{N-1}$ as $Z^{N-1}\left(a \mid a^{*}, T\right)$, where $Z\left(a \mid a^{*}, T\right)$ is defined in (1).

${ }^{21}$ Depending on parameters, it is possible to have $c^{*}>\frac{\lambda}{N}$, in which case, part (1) of Proposition 3 is irrelevant. To be specific, one can verify that $\lim _{T \rightarrow \infty} c^{*}=\frac{\lambda}{N}, \lim _{T \rightarrow 0} c^{*}=0$, and for $N \geq 3$, there is a unique $T^{*}$ such that $c^{*}=\frac{\lambda}{N}$ when $T=T^{*}$.
} 
2. If $c \in\left(0, \min \left\{c^{*}, \frac{\lambda}{N}\right\}\right]$, all agents search with an interior cutoff $a^{*} \in(0,1)$ such that

$$
\lambda \int_{a^{*}}^{1}\left[Z^{N-1}\left(x \mid a^{*}, T\right)-Z^{N-1}\left(a^{*} \mid a^{*}, T\right)\right] d F(x)=c .
$$

The intuition for Proposition 3 is similar as before: In the unique symmetric equilibrium, each agent searches with the minimal stationary cutoff 0 when the search cost $c$ is relatively big, and each agent searches with a stationary interior cutoff when the search cost $c$ is relatively small. One can again formulate the parameter conditions in Proposition 3 in terms of the contest duration, in that all agents search with an interior cutoff value if $T$ is sufficiently large, and search with the minimal cutoff value $a^{*}=0$ otherwise.

\subsubsection{The Public Contest}

Now consider the $N$-agent public contest. A useful observation is that while the equilibrium analysis for this contest is complicated by the need to evaluate the decisions of all agents after various histories, the public contest admits a convenient recursive-like structure in that after each submission, the remaining agents face a similar but smaller public contest game. The whole game hence unfolds over time like a set of nesting Russian dolls: Although each submission might modify the (continuation) cutoffs employed by the remaining agents, the analysis of each remaining agent's decision is isomorphic to the original contest game, but with a shorter horizon and fewer opponents. We will heavily rely on this 'recursive' structure in our characterization in the sequel. ${ }^{22}$

First, as before, if at any point of the game an agent submits a lucky draw with an innovation at least $\hat{a}=F^{-1}\left(1-\frac{c}{\lambda}\right)$, the submission will forestall further search from all the other agents, winning the contest.

Next, we analyze a class of auxiliary contests with different numbers of competing agents. Our analysis here identifies crucial cutoff values that will be useful for the original public contest. Consider a public contest with $(N-K)$ agents where $K \in\{0, \ldots, N-2\}$ after a history of no previous submissions. ${ }^{23}$ The following lemma characterizes the cutoff values $\alpha_{t}^{*}(K)$, which will take a similar role as $\alpha_{t}^{*}$ in (5) for Proposition 2.

\footnotetext{
${ }^{22}$ Notice that such a convenient structure arises in our continuous-time contest since submissions from the agents occur sequentially (and multiple submissions at the same instant can be ignored throughout).

${ }^{23}$ The auxiliary $(N-K)$-agent public contest corresponds to a history in the original $N$-agent public contest where $K$ agents have submitted their innovations. The case with $K=N-1$ is 'degenerate' in that the remaining single agent will search with the highest submitted innovation as his cutoff.
} 
Lemma 1 Consider an auxiliary public contest with $(N-K)$ agents. In the unique symmetric equilibrium, each agent adopts a unique cutoff $\alpha_{t}^{*}(K)$ after a history of no previous submissions, where for $t \in[0, T)$

i. $\alpha_{t}^{*}(K)=0$ if

$$
\frac{e^{(N-K-1)(\lambda-c)(T-t)}-1}{(N-K-1)(T-t)} \leq \lambda
$$

ii. $\alpha_{t}^{*}(K) \in(0, \hat{a})$ otherwise and is the unique scalar solving

$$
\frac{e^{\lambda(N-K-1)\left[F(\hat{a})-F\left(\alpha_{t}^{*}(K)\right)\right](T-t)}-1}{(N-K-1)(T-t)}=\lambda\left[1-F\left(\alpha_{t}^{*}(K)\right)\right] .
$$

In addition, for an interior $\alpha_{t}^{*}(K)$, we have $\frac{d \alpha_{t}^{*}(K)}{d t}<0, \frac{d \alpha_{t}^{*}(K)}{d K}<0, \frac{d \alpha_{0}^{*}(K)}{d T}>0$, and $\lim _{T \rightarrow \infty} \alpha_{0}^{*}(K)=\hat{a}=F^{-1}\left(1-\frac{c}{\lambda}\right)$.

Lemma 1 is a generalization of the equilibrium cutoff function $\alpha_{t}^{*}$ (after a history of no submission) of the two-agent public contest identified in Proposition 2. The intuition of Lemma 1 follows that of Proposition 2: If $T$ is small or $c$ is large, then we have the minimal cutoff value of $\alpha_{t}^{*}(K)=0$ for all $t$. Otherwise, all agents adopt a cutoff value that is interior but decreasing over time.

While Lemma 1 is obtained for the auxiliary public contests with $(N-K)$ agents, the cutoffs $\alpha_{t}^{*}(K)$ actually serve as crucial benchmark cutoffs that will determine stopping decisions for all non-submitting agents in the $N$-agent public contest. To illustrate, consider a history in the $N$-agent public contest at $t$ where $K$ agents have submitted their innovations before $t$, with the best submission being $a(K)$ among the $K$ submissions. If $a(K) \leq \alpha_{t}^{*}(K)$, the $K$ submitted values are then not binding in the sense that the remaining $(N-K)$ agents can treat the remainder of the contest as one with $(N-K)$ agents after a history of no submissions and adopt the higher cutoff $\alpha_{t}^{*}(K)$ at the instant t. However, if $a(K) \in\left(\alpha_{t}^{*}(K), \hat{a}\right)$, the remaining agents will 'update' their behavior based on the new information and will then search with $a(K)$ being the binding cutoff at $t$ - the agents might search with a cutoff higher than $a(K)$ in the future after yet another (better) submission from one of the $(N-K)$ agent, and so on. The above illustration of the agents' searching behavior is formally addressed in our next proposition, which completely characterizes the symmetric equilibrium of the $N$-agent public contest. The cutoff $\alpha_{t}^{*}(K)$ in Proposition 4 is defined in Lemma 1.

Proposition 4 (Public Contest with $N$ Agents) The $N$-agent public contest with $T<$ $\infty$ admits a unique symmetric equilibrium where at any $t \in[0, T)$, 
1. after any history, if an agent submits an innovation with value $a \geq \hat{a}=F^{-1}\left(1-\frac{c}{\lambda}\right)$, the remaining agents (if any) stop searching immediately and submit their respective best innovations;

2. after a history with exactly $(N-1)$ submissions and the highest submitted innovation being $a^{\prime}<\hat{a}$, the remaining agent searches with cutoff $a^{\prime}$;

3. after a history with exactly $K$ submissions, $K \in\{0, \ldots, N-2\}$, with the highest submitted innovation being $a(K)<\hat{a}$, the remaining agents search with cutoff $\max \left\{a(K), \alpha_{t}^{*}(K)\right\}$, where we impose $a(K)=0$ if $K=0$, i.e., the cutoff is $\alpha_{t}^{*}(0)$ when $K=0$, or when there is no submission.

Hence, the symmetric equilibrium in the $N$-agent public contest, while more complicated, is structurally identical to that in the two-agent public contest: The agents search with a time-decreasing cutoff $\alpha_{t}^{*}(0)$ after a history of no submissions, and after observing a submission, the remaining agents respond by revising their common cutoffs strategically given the submitted innovation.

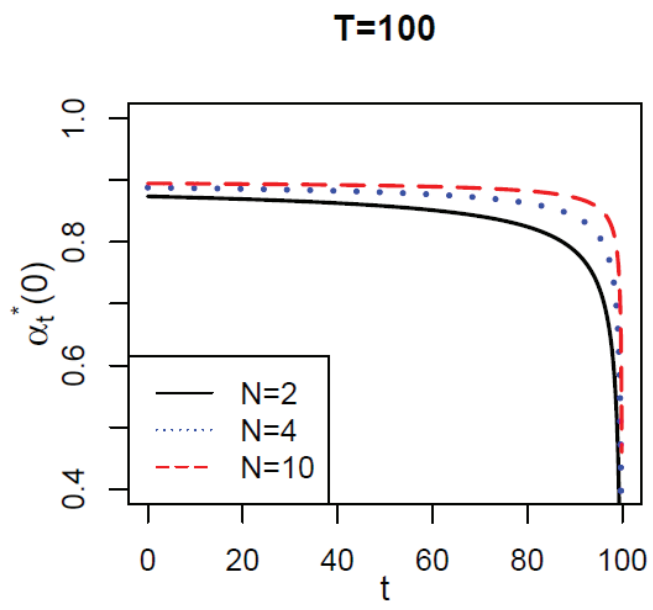

Figure 2. Equilibrium Cutoff $\alpha_{t}^{*}(0)$ with $\lambda=1, c=0.1, T=100$, and $F$ uniform.

Figure 2 presents a graphical illustration of $\alpha_{t}^{*}(0)$ for $N \in\{2,4,10\}$, which is indeed strictly decreasing in $t$. In addition, observe that the cutoff $\alpha_{t}^{*}(0)$ strictly increases in $N$. This stands in sharp contrast with the $N$-agent hidden contest, where the timeinvariant equilibrium cutoff $a^{*}$ in Proposition 3 is strictly decreasing in $N{ }^{24}$ Both the comparative statics result from more competition when there are more agents. ${ }^{25}$ However,

\footnotetext{
${ }^{24}$ The equilibrium cutoffs $a^{*}$ in Proposition 3 given the parameters $(\lambda=1, c=0.1$, and $F$ uniform) for the three cases are respectively $a^{*}(N=2)=0.8, a^{*}(N=4)=0.6$, and $a^{*}(N=10)=0$.

${ }^{25}$ Such comparative statics can be shown explicitly using Proposition 3 and Proposition 4 , though the
} 
the uncertainty resulting from the hidden policy (of not knowing the opponents' states) implies that the $N$ agents will in expectation split the prize in equilibrium. Hence, more competition implies a smaller share of prize for each agent, and each agent responds by searching with a lower cutoff. In the public contest, more competition manifests itself in a higher equilibrium cutoff, since the agents always 'observe' some information about the opponents and the dominant incentive on the equilibrium path is to obtain a high innovation in order to preempt the opponents. We will come back to this issue when we compare the public policy and the hidden policy in Section 4.

\section{Ranking Disclosure Policies}

We now analyze how the sponsor should disclose agents' submissions over time in the research contest. Specifically, we will consider more general disclosure policies and rank these disclosure policies from the sponsor's point of view.

Our previous equilibrium analysis indicates that a submitted innovation from an agent, if revealed in the middle of the contest, can affect cutoff values for the remaining agents' search for the rest of the contest. Hence ex ante, a disclosure policy can generate correlation in the agents' equilibrium search behavior, which makes the calculation of the sponsor's expected payoffs from different disclosure policies rather complex. As a result, a full-blown comparison of various disclosure policies for a finite-horizon contest is in general intractable. To facilitate a clean and general characterization, we compare various disclosure policies for the sponsor in the limiting case of infinite-horizon contests. ${ }^{26}$

In an infinite-horizon contest, the agents are allowed to search for indefinite duration. It is true that for many contests in practice, even the most far-sighted sponsors would typically organize finite-horizon innovation contests. But for society as a whole, it may be preferable (and there are good reasons) for governments and big corporations to procure important innovations with indefinite duration. Indeed, there are real-world contests that can be approximately regarded as contests of indefinite duration, such as prizes for various unsolved mathematical and medical problems. ${ }^{27}$

calculation will be somewhat cumbersome. We will come back to the issue of comparative statics in $N$ when we analyze infinite-horizon contests, where the analysis is much cleaner.

${ }^{26}$ We will nevertheless analyze the principal's disclosure incentives for short horizons in Section 5. As we will see, the comparison of different disclosure policies for short-horizon contests indeed depends crucially on the functional forms of $v(\cdot)$ and $F(\cdot)$, confirming that general results on disclosure for finite-horizon contests are typically difficult.

${ }^{27}$ Examples of this sort include Deloitte's Cancer XPRIZE and the 7 Millennium Prize Problems established by the Clay Mathematics Institute. Infinite-horizon contests have also been discussed in 


\subsection{General Disclosure Policies}

We first define a general information disclosure policy. ${ }^{28}$ Recall that the sponsor's information or history at $t$ is $\left(\mathbf{s}_{t}\right)_{\tau \leq t}$ where $\mathbf{s}_{t}=\left(s_{1, t}, \ldots, s_{n, t}\right)$ is the contest submission status at $t$ and $s_{i, t} \in\left\{\varnothing,\left(\tau_{i}, \omega_{i}\right)\right\}$ is $i$ 's submission status at $t$, i.e., either $i$ has not submitted up to $t\left(s_{i, t}=\varnothing\right)$ or submitted his innovation with value $\omega_{i}$ at $\tau_{i} \leq t\left(s_{i, t}=\left(\tau_{i}, \omega_{i}\right)\right)$. A disclosure policy $D$ is a sequence of measurable functions $\psi_{t}$ mapping from the set of all possible histories of the sponsor up to $t$ to a set of messages, $\psi_{t}: S_{t} \rightarrow M_{t}$, where $S_{t}$ is the set of all possible $\mathbf{s}_{t}$ and $M_{t}$ is a measurable message space. We will consider disclosure policies that satisfy several natural restrictions: (i) the sponsor can only withhold information (to various degrees), but cannot fabricate information, ${ }^{29}$ (ii) disclosure is public so that remaining agents are symmetrically informed about the disclosed information, (iii) disclosure is anonymous in that whether to disclose a piece of information does not depend on the identity of submitting agents, i.e., for $\mathbf{s}_{t} \in S_{t}$ and any permutation of $\mathbf{s}_{t}, \mathbf{s}_{t}^{\pi}$, we have $\psi_{t}\left(\mathbf{s}_{t}\right)=\psi_{t}\left(\mathbf{s}_{t}^{\pi}\right)$. Notice the requirements (ii) and (iii) are important for our focus on symmetric equilibria. Finally, recall that the sponsor commits publicly to a disclosure policy and cannot modify the disclosure policy afterwards.

In words, a disclosure policy specifies how the sponsor should dynamically disclose information about submissions. Examples of such policies include the previous public policy, in which the sponsor discloses $\mathbf{s}_{t}$ in real time, and the hidden policy where no information is disclosed until the end of the contest. Alternatively, the sponsor can disclose any descriptive statistics that summarizes the submitted innovations at any point of the contest. These examples demonstrate that, even with the above restrictions (i)-(iii), the set of feasible disclosure policies is very large. We hence take a more modest approach: We consider instead a restricted but natural set of simple disclosure policies. After characterizing the corresponding symmetric equilibrium outcomes for these disclosure policies, we then provide a complete ranking result for the set of simple disclosure policies. We discuss in the end the generality of this complete ranking result in helping us understand how the sponsor fares under various disclosure policies.

\footnotetext{
Taylor (1995)[23] (page 881).

${ }^{28}$ Though we will not be able to identify optimal disclosure policies among all such general disclosure policies, a clear definition of these general disclosure policies will provide a useful overall view on the set of disclosure policies that can be appropriately studied in our setting.

${ }^{29}$ In particular, the principal cannot disclose more positive submissions than she has received. This is a sensible restriction given that all submissions can typically be corroborated by submitted agents.
} 


\subsection{Simple Disclosure Policies}

To motivate the set of simple disclosure policies, we first present Proposition 5 for the infinite-horizon hidden and public contests.

Proposition 5 (Infinite-Horizon Public and Hidden Contests) Consider the N-agent contest games in Section 3.3 but with infinite horizons.

1. In the hidden contest, there is a unique pure-strategy symmetric equilibrium where all the agents search with cutoff $a^{H}=F^{-1}\left(1-\frac{N c}{\lambda}\right)$.

2. In the public contest, there is a unique pure-strategy symmetric equilibrium where

(a) All the agents search with cutoff $\hat{a}=F^{-1}\left(1-\frac{c}{\lambda}\right)$ when there is no previous submission, or when there are $(N-2)$ or less submissions with the best submitted value $a^{\prime}$ being $a^{\prime}<\hat{a}$. If there are $(N-1)$ submissions with the best submitted value $a^{\prime}$ being $a^{\prime}<\hat{a}$, the remaining agent searches with cutoff $a^{\prime}$.

(b) If an agent submits an innovation $a \geq \hat{a}$ at some $t$, the remaining agents stop searching (and submit their respective best innovations) immediately.

Proposition 5 is a direct consequence of Proposition 3 and Proposition 4, and the symmetric equilibria are respectively the limiting points of the symmetric equilibria of the corresponding finite-horizon games in Section 3.3. ${ }^{30}$ In the symmetric equilibrium of the hidden contest, every agent stops searching after passing an identical cutoff of $a^{H}$, while in the symmetric equilibrium of the public contest, generically there is exactly one agent whose submission passes the cutoff $\hat{a}$, since upon seeing this submission, all the remaining $(N-1)$ agents stop and submit their respective best innovations (which are all below $\hat{a}$ ). In particular, notice that all agents adopt a constant and common cutoff on the equilibrium path in both the hidden and the public contest.

We now consider a set of simple disclosure policies that (1) encompasses the public and the hidden disclosure policies as special cases and (2) generates qualitatively similar symmetric equilibrium outcomes as those in Proposition 5.

Definition 1 For $k \in\{1, \ldots, N\}$, let $\left\{m_{1}, \ldots, m_{k}\right\} \subseteq\{1, \ldots, N\}$ be an arbitrary set of exactly $k$ agents, and $a^{k}=F^{-1}\left(1-\frac{k c}{\lambda}\right)$ be a cutoff. Consider a contest submission status $\mathbf{s}_{t}^{k}$ at $t$ where for each $n \in\left\{m_{1}, \ldots, m_{k}\right\}, s_{n, t}^{k}=\left(\tau_{n}, \omega_{n}\right)$ with $\tau_{n} \leq t$ and $\omega_{n} \geq a^{k}$, and

\footnotetext{
${ }^{30}$ Since it is a special case of Proposition 6, we omit the proof of Proposition 5.
} 
for each $i \in\{1, \ldots, N\} \backslash\left\{m_{1}, \ldots, m_{k}\right\}, s_{i, t}^{k}=\varnothing$. A simple $k$-disclosure policy is one where the sponsor announces "there are exactly $k$ submissions each passing the cutoff $a^{k}$ " at time $t$ whenever $\mathbf{s}_{t}=\mathbf{s}_{t}^{k}$, and the sponsor remains silent whenever $\mathbf{s}_{t} \neq \mathbf{s}_{t}^{k}$.

Hence, with a simple $k$-disclosure policy, the sponsor only sends out exactly one public message in the contest, confirming whether there have already been exactly $k$ submissions, each (weakly) passing the cutoff $a^{k}$. Here $a^{k}$ is specifically chosen so that all the remaining $(N-k)$ agents stop searching and submit their respective highest innovations (which are all below $a^{k}$ ) after receiving the public message in equilibrium. Proposition 6 characterizes the unique symmetric equilibrium for each simple $k$-disclosure policy:

Proposition 6 (Symmetric Equilibria for Simple Disclosure Policies) Consider the infinite-horizon $N$-agent contest game with a simple $k$-disclosure policy. There is a unique pure-strategy symmetric equilibrium where

(i) Absent an announcement from the sponsor, each agent searches with the cutoff $a^{k}=$ $F^{-1}\left(1-\frac{k c}{\lambda}\right)$ and submits his best innovation whenever it is above $a^{k}$.

(ii) Upon receiving the sponsor's announcement, the remaining agents stop searching immediately (and submit their respective best innovations).

Notice that according to Proposition 6, the simple 1-disclosure policy generates the same equilibrium outcome as in the public contest, while the simple $N$-disclosure policy leads to the same equilibrium outcome as in the hidden contest, both presented in Proposition 5 .

\subsection{Ranking Simple Disclosure Policies}

The characterization of the unique symmetric equilibrium for each simple disclosure policies facilitates the comparison of the simple disclosure policies. In particular, our main result of the paper, Proposition 7 shows that the sponsor ranks the corresponding $N$ simple $k$-disclosure policies strictly.

Proposition 7 (Ranking Simple Disclosure Policies) Consider the infinite-horizon $N$-agent contest under the $N$ simple disclosure policies. For $\ell \in\{1, \ldots, N-1\}$, the sponsor strictly prefers the simple $\ell$-disclosure policy to the simple $(\ell+1)$-disclosure policy. 
Given that the simple 1-disclosure policy (resp., the simple $N$-disclosure policy) is outcome equivalent to the public policy (resp., the hidden policy), Proposition 7 immediately implies that the public policy is strictly better than the hidden policy for the sponsor:

Corollary 1 In the infinite-horizon $N$-agent contest game, the sponsor strictly prefers the public policy to the hidden policy. In addition, the payoff difference between the public policy and the hidden policy for the sponsor is strictly increasing in $N$.

A main message from Proposition 7 (and Corollary 1) is that the sponsor can induce different search behavior and hence different equilibrium outcomes only by varying the disclosure of submission information. The intuition of Proposition 7 hinges on the fact that an agent faces different degrees of uncertainty about his chance of winning the prize under different simple disclosure policies, which generates different search behavior and different outcomes for the sponsor. This is reminiscent of the information effect discussed earlier: Under the simple 1-disclosure or the public policy, each agent knows that once he submits an innovation above $a^{1}=\hat{a}$, the submission is revealed publicly, which guarantees winning. However, under the simple $N$-disclosure or the hidden policy, an agent knows nothing about the others' submission status and the uncertainty that some other agent may have already found a very high innovation holds the agent back from searching aggressively. Proposition 7 also fully delineates all the 'intermediate' cases, with the cutoffs $a^{k}$ designed so that all the remaining agents stop searching upon seeing the message from the sponsor. Notice that, while the intuition of Proposition 7 is that agents search more aggressively under a simple $k$-disclosure policy than under a simple $(k+1)$-disclosure policy, the result is more subtle: Although $a^{k}>a^{k+1}$, there is one more submission above $a^{k+1}$ under the simple $(k+1)$-disclosure policy. Hence the sponsor strictly prefers less random draws with a higher cutoff to more random draws but with a lower cutoff, given that the sponsor value is strictly increasing in the best innovation.

Finally, for the comparative statics result in Corollary 1, notice that in the public contest, the cutoff $\hat{a}=F^{-1}\left(1-\frac{c}{\lambda}\right)$ is independent of $N$, while the cutoff in the hidden contest $a^{H}=F^{-1}\left(1-\frac{N c}{\lambda}\right)$ is strictly decreasing in the number of agents in the contest. This phenomenon is similar to that for finite-horizon contests and has been documented in Figure 2. The comparative statics is again driven by information: In the hidden contest, agents receive no information about the opponents and a larger $N$ implies that more agents split the grand prize, leading to a lower equilibrium cutoff. But in the public contest, a submission with innovation $\hat{a}$ or higher will stop the opponents from searching, and a larger $N$ does not change this preemptive incentive. 


\subsection{Generality of Proposition 7}

Our ranking result in Proposition 7 goes beyond the set of simple disclosure policies. To begin with, while the sponsor can choose various disclosure policies, ultimately what she cares about is the equilibrium outcome a disclosure policy induces. We now argue, somewhat informally, that our ranking result in Proposition 7 is 'complete' if we restrict attention to disclosure policies that generate symmetric equilibria in stopping strategies.

Consider the set of all symmetric equilibria in stopping strategies. ${ }^{31}$ First individual rationality and the fact that an agent always incurs cost before submission jointly imply that all the agents stop searching (hence making submissions) in finite time with probability one in any equilibrium. In addition, though there is only one winner ex post in any equilibrium, the contest game effectively ends for the agents at the submission stage which is the stage after all agents have submitted their best innovations but before the sponsor announces the winner. The reason is that after the submission stage, the sponsor simply announces the winner with the best innovation, with no strategic choice from the agents. It is hence sufficient to consider symmetric equilibrium outcomes at the submission stage. For a given symmetric equilibrium, ex ante (i.e., viewed before the contest begins), there are at least one submission and at most $N$ submissions that can win the prize with positive probabilities at the submission stage, regardless of the sponsor's disclosure policy. ${ }^{32}$ We say that a symmetric equilibrium is one with $k \in\{1, \ldots, N\}$ effective submissions if from an ex ante point of view there are exactly $k$ submissions that can win the grand prize with strictly positive probability at the submission stage. ${ }^{33}$

Given the above definition, Proposition 6 implies that a simple $k$-disclosure policy induces a unique symmetric equilibrium with exactly $k$ effective submissions. Hence our set of simple disclosure policies can induce the entire set of symmetric equilibria with (deterministic) numbers of effective submissions. Importantly, observe that although a symmetric equilibrium outcome with $k$ effective submissions can be generated by various disclosure policies, a simple $k$-disclosure policy defines the common and key equilibrium threshold $a^{k}=F^{-1}\left(1-\frac{k c}{\lambda}\right)$ for all agents in a symmetric equilibrium in stopping strategies with $k$ effective submissions. To see this, in the unique symmetric equilibrium induced by the

\footnotetext{
${ }^{31}$ More specifically, we consider symmetric and pure Markov perfect equilibria in stopping strategies, where agents' strategies do not depend on payoff irrelevant events, such as the identities of agents who have submitted, and past submissions with innovations that are not the highest (due to our winner-takesall payment scheme), etc.

${ }^{32}$ Due to symmetry, if there are $m>1$ submissions that win the prize with positive probability, then each of $m$ submissions wins the prize with probability $\frac{1}{m}$, from an ex ante point of view.

${ }^{33}$ We will address later the possibility that an equilibrium outcome is a probability distribution over the $(N)$ symmetric equilibrium outcomes with various effective submissions.
} 
simple $k$-disclosure policy, $k \in\{1, \ldots, N\}$, each agent's instantaneous gain from searching at each instant on the equilibrium path is

$$
\lambda \int_{a^{k}}^{1}\left[\frac{F(a)-F\left(a^{k}\right)}{1-F\left(a^{k}\right)}\right]^{k-1} d F(a)-c=\frac{\lambda\left[1-F\left(a^{k}\right)\right]}{k}-c=0 .
$$

Hence, each agent's equilibrium payoff is identically zero. While a direct consequence of our contest having an infinite horizon, this result implies that each agent is fully 'exploited,' in that each agent's individual rationality condition is binding in each of the symmetric equilibria, and any symmetric equilibrium outcome with $k$ effective submissions and an equilibrium cutoff strictly higher than $a^{k}=F^{-1}\left(1-\frac{k c}{\lambda}\right)$ would necessarily lead to a negative equilibrium payoff, which is impossible.

Proposition 7 is stated as a ranking result for the $N$ simple disclosure policies. However, what it actually ranks is the set of symmetric equilibria with various numbers of effective submissions. It implies that the sponsor strictly ranks the $N$ symmetric equilibrium outcomes and the symmetric equilibrium outcome with 1 effective submission leads to the best expected payoff and the symmetric equilibrium outcome with $N$ effective submissions yields the worst expected payoff for the sponsor. This alternative interpretation of Proposition 7 enables us to compare various disclosure policies based on the symmetric equilibria in stopping strategies induced by these policies. Indeed this is exactly how we derived Corollary 1: By Proposition 5, the public contest induces a unique symmetric equilibrium with 1 effective submission, while the hidden contest induces a unique symmetric equilibrium with $N$ effective submissions. Proposition 7 hence implies that the public policy is strictly better than the hidden policy.

Finally, Proposition 7 only compares symmetric equilibrium outcomes with a deterministic number of effective submissions. Ranking disclosure policies that induce symmetric equilibrium outcomes with random numbers of effective submissions, if any, is slightly more complicated, given that we impose no restriction on the sponsor's risk attitude (recall that we only assume that the sponsor's valuation $v(a)$ is strictly increasing in $a$ ). However, given that the sponsor ranks the symmetric equilibrium outcomes with $k$ effective (deterministic) submissions strictly, such disclosure policies cannot be better than the public policy and cannot be worse than the hidden policy. 


\section{Discussion}

\subsection{Short Horizons}

We have hinted in the discussion after Figure 1 that when the contest horizon is short, the agents may ex ante search more intensively in the hidden contest than that in the public contest. A definitive comparison between the public and hidden disclosure policies for the sponsor is difficult to obtain, due to (again) the agents' correlated equilibrium search behavior in the public contest, making it difficult to derive the probability distribution of the winning innovation. Nevertheless, we now show explicitly that a hidden contest can strictly outperform a public contest in a short-horizon contest.

From an intuitive point of view, the sponsor can strictly prefer the hidden policy for two reasons: First, in the public contest, the agents search with a decreasing cutoff in equilibrium after a history of no submission, and this equilibrium cutoff can drop to zero near the deadline, as demonstrated in Proposition 2 and Figure $1 .^{34}$ Hence, while no information is always good information for the agents in a public contest, the agents' incentives to search are dampened after observing no previous submission in the public contest, which can be counter-productive for the sponsor. In comparison, the agents' search intensity always "remains the same" throughout in the hidden contest. Second, and perhaps more importantly, there can be at most one innovation above $\hat{a}=F^{-1}\left(1-\frac{c}{\lambda}\right)$ in the public contest, since a submission with an innovation $\hat{a}$ or above immediately stops the other agents from searching. However, with no submission information ever being released, there can be multiple innovations above $\hat{a}$ in the end in the hidden contest. Hence, a hidden contest can be strictly better if the sponsor attaches high valuations only to sufficiently good innovations.

We now present a possibility result (Proposition 8), which demonstrates an explicit and complete example where the sponsor strictly prefers the hidden contest.

Proposition 8 (Short Horizons) With a short contest horizon, the hidden disclosure policy can strictly outperform the public contest for the sponsor.

Consider a two-agent contest where $F$ is the uniform distribution, $c=0.4, \lambda=1$, and

\footnotetext{
${ }^{34}$ The phenomenon that the equilibrium cutoff can drop to the lowest cutoff of 0 in the public contest is however driven by our modeling choice of Poisson arrival of innovation opportunities, since given a finite horizon $T$, there is always a positive probability that an agent's opponents have received no innovation opportunities. We thank Curt Taylor for bringing this to our attention.
} 
contest horizon $T \leq 1$. The sponsor's valuation for the innovation $v(a)$ is such that

$$
v(a)=\left\{\begin{array}{l}
0, \text { if } a \leq x \\
1, \text { if } a>x
\end{array} \text { where } x \in(\hat{a}, 1)\right.
$$

Hence the sponsor only cares about sufficiently good innovations. Since $c<\frac{\lambda\left(1-e^{-\lambda T}\right)}{2}$ and $e^{(\lambda-c)(T-t)}-1 \leq \lambda(T-t)$ for all $t \in[0,1]$, the equilibrium cutoff is " 0 " in the hidden contest and both agents start searching with cutoff "0" as well in equilibrium in the public contest. Notice that $\hat{a}=F^{-1}\left(1-\frac{c}{\lambda}\right)=0.6$ and according to $v(a)$, it suffices to calculate probability distribution of winning innovations that are above $\hat{a}=0.6$.

Ignoring the contest prize throughout, the sponsor's expected payoff from the hidden contest is

$$
\begin{aligned}
\mathbb{E} V^{H}(x) & =P_{1}\left[1-\frac{x-\hat{a}}{1-\hat{a}}\right]+P_{2}\left[1-\left(\frac{x-\hat{a}}{1-\hat{a}}\right)^{2}\right] \\
P_{1} & =2\left(1-e^{-T}\right)(1-\hat{a})\left[e^{-T}+\left(1-e^{-T}\right) \hat{a}\right] \text { and } P_{2}=\left[\left(1-e^{-T}\right)(1-\hat{a})\right]^{2},
\end{aligned}
$$

where $P_{1}$ is the probability that exactly one submission is above $\hat{a}$ and $P_{2}$ is the probability that both submissions are above $\hat{a}$ in equilibrium. ${ }^{35}$ The sponsor's expected payoff from the public contest can be calculated as:

$$
\begin{aligned}
& \mathbb{E} V^{P}(x)=\operatorname{Pr}(\text { win })\left[1-\frac{x-\hat{a}}{1-\hat{a}}\right] \text { where } \\
& \operatorname{Pr}(\text { win })=1-e^{-2 T}-\int_{0}^{T} 2 e^{-2 t} \int_{0}^{\widehat{a}}\left[e^{-(T-t)(1-a)}+\left(1-e^{-(T-t)(1-a)}\right) \frac{\widehat{a}-a}{1-a}\right] d a d t .
\end{aligned}
$$

Here $\operatorname{Pr}($ win) is the probability that the winning innovation is above $\hat{a}$ and the third term of $\operatorname{Pr}($ win) is the probability that both agents' submitted innovations are below $\hat{a}$, with at least one agent obtaining an innovation above 0 .

The comparison between $\mathbb{E} V^{H}(x)$ and $\mathbb{E} V^{P}(x)$ is presented in Figure 3, which demonstrates that the hidden contest generates strictly higher expected payoff than the public contest. At first sight, it seems puzzling to have $\mathbb{E} V^{H}(x)>\mathbb{E} V^{P}(x)$ even in this simple example: In the hidden contest, both agents only search with cutoff 0 in equilibrium. However, in the equilibrium of the public contest, while both agents also start searching with cutoff 0 , it can happen that an agent searches with a cutoff strictly higher than 0

\footnotetext{
${ }^{35}$ For the other two terms in $E V^{H}(x)$, recall that according to $v(a)$, an innovation is worth 1 to the sponsor only if the innovation is above $x$.
} 
after his opponent obtains and submits a non-zero innovation. Hence, overall the agents seem to search more intensively in the public contest. The wedge between $\mathbb{E} V^{H}(x)$ and $\mathbb{E} V^{P}(x)$ in Figure 3 comes from the possible number of submissions above $\hat{a}$ : In the public contest, once an agent submits an innovation above $\hat{a}$, the other agent stops immediately. There is however a positive probability that both agents submit innovations above $\hat{a}$ in the hidden contest, which is particularly important if $x$ is large here (i.e., the sponsor only values sufficiently good innovations). This is the key driving force of our result $\mathbb{E} V^{H}(x)>\mathbb{E} V^{P}(x){ }^{36}$

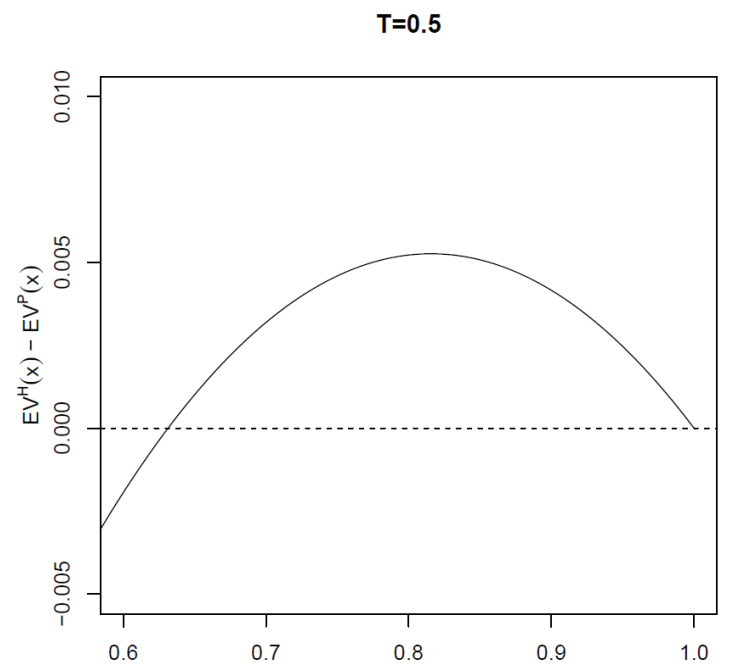

Figure 3. Comparing the Hidden and Public Contests with a Short Horizon.

\subsection{Strategic Delay}

Our analysis so far has focused on scenarios where the cost $c$ is both the fiduciary and opportunity cost for searching for an innovation. In these scenarios, an agent submits his best innovation whenever he stops searching, and strategic delay, i.e., intentionally holding the best innovation without searching and waiting to submit it later, is always suboptimal and cannot arise in equilibrium. As we have mentioned (in Remark 1), this assumption can be strong in some contests, such as the Netflix prize, where the opportunity cost of remaining in the contest without searching is most likely negligible. And in such contests, holding an innovation without submitting does not incur additional cost, except perhaps running the risk of being preempted by an opponent. ${ }^{37}$

\footnotetext{
${ }^{36}$ It is clear though that the step-function specification in (12), while simplifies our calculation, is not necessary in driving $\mathbb{E} V_{H}(x)>\mathbb{E} V_{P}(x)$.

${ }^{37}$ Indeed, BellKor Pragmatic Chaos from AT\&T won the Netflix prize of one million dollars in September of 2009. The other forerunner contestant, the Ensemble, although had matched BellKor's winning
} 
We consider here an alternative setting where $c$ is only the fiduciary (search) cost. Hence, an agent has the option of stopping and waiting for a time to submit, without incurring any cost. Intuitively, an agent now indeed has incentives to delay submission in the public contest: After a history with no submissions, if an agent submits an innovation before $T$, the innovation is revealed to his opponent, (potentially) prompting the opponent to search further, which can be suboptimal if there is ample time left before the deadline $T$. On the other hand, holding an innovation without submitting it, which is costless, also allows an agent to search further after a submission from his opponent, as long as the opponent's submission is not preemptively high.

We now construct a symmetric equilibrium featuring strategic delay in a two-agent public contest with deadline $T$ and no cost from stopping and waiting in the contest. ${ }^{38}$ In equilibrium, each agent starts searching with a time-independent cutoff $\bar{a} \in[0, \hat{a})$. After a history of no submission, an agent makes a submission if he obtains an innovation above $\hat{a}$, winning the prize. If however the agent obtains an innovation in $(\bar{a}, \hat{a})$, the agent then stops searching and waits without submitting, until time $T$ or a submission from his opponent, whichever occurs earlier. If the opponent submits $a^{\prime}$ before $T$, the agent then either searches with $a^{\prime}$ as the new cutoff $\left(a^{\prime}<\hat{a}\right)$ or drops out $\left(a^{\prime} \geq \hat{a}\right)$.

As before, we analyze the agents' incentives after different histories. First, it is easy to see that the agents' incentives are the same as in Proposition 2 when an agent submits an innovation: An innovation above $\hat{a}$ stops the opponent from searching and wins the prize, while an innovation $a$ below $\hat{a}$ induces the opponent searches with $a$ being the new cutoff. So we focus on histories where no submission has been made up to some $t$. Let agent $i$ 's best innovation at $t$ be $a_{t} \in[0, \hat{a})$. Denote the agent's continuation payoff from stopping at $t$ and waiting to submit $a_{t}$ at $T$ as $V_{t}\left(a_{t}\right)$. Given the winner-takes-all payoff structure, we have, on the equilibrium path,

$$
V_{t}\left(a_{t}\right)=\operatorname{Pr}\left(a_{T}^{-i}<a_{t} \mid a_{t}^{-i}<\hat{a}\right)=\frac{\operatorname{Pr}\left(a_{t}^{-i}<\hat{a} \mid a_{T}^{-i}<a_{t}\right) \operatorname{Pr}\left(a_{T}^{-i}<a_{t}\right)}{\operatorname{Pr}\left(a_{t}^{-i}<\hat{a}\right)}=\operatorname{Pr}\left(a_{T}^{-i}<a_{t}\right),
$$

where $\operatorname{Pr}\left(a_{t}^{-i}<\hat{a} \mid a_{T}^{-i}<a_{t}\right)=1$ and $\operatorname{Pr}\left(a_{t}^{-i}<\hat{a}\right)=1$ given that the opponent has not submitted at $t$. In other words, given that $a_{t}^{-i}<\hat{a}$, agent $i$ only wins the prize when agent $-i$ submits at/before $T$, with an eventual innovation $a_{T}^{-i}$ less than $a_{t}$. Agent $i$ 's

score, submitted their result 20 minutes late, and "that 20 minutes was worth a million dollars." (The Netflix Prize: How a \$1 Million Coding Contest Changed Streaming, Thrillist, 09/03/2018).

${ }^{38}$ For expositional clarity, we only present the result for a two-agent public contest. A similar result can be obtained for a general $N$-agent public contest. The result and proof for the general contest are available upon request. 
instantaneous gain from searching for the next instant and then stopping is

$$
\lambda \int_{a_{t}}^{1}\left[V_{t}(a)-V_{t}\left(a_{t}\right)\right] d F(a)-c .
$$

Since $V_{t}\left(a_{t}\right)$ is strictly increasing in $a_{t}$ and hence the above instantaneous gain is strictly decreasing in $a_{t}$, there is at most one value $\bar{a}$, such that agent $i$ is exactly indifferent between searching and stopping at the cutoff $\bar{a}$, i.e.,

$$
\lambda \int_{\bar{a}}^{1}\left[V_{t}(a)-V_{t}(\bar{a})\right] d F(a)=c .
$$

And we again impose $\bar{a}=0$ if there is no value of $\bar{a}$ in $[0,1]$ solving (13).

Since after a history of no submission, $V_{t}\left(a_{t}\right)=1$ if $a_{t}>\hat{a}$, we rewrite (13) as

$$
\lambda \int_{\bar{a}}^{\hat{a}}\left[V_{t}(a)-V_{t}(\bar{a})\right] d F(a)+\lambda \int_{\hat{a}}^{1}\left[1-V_{t}(\bar{a})\right] d F(a)=c,
$$

which after rearranging the terms and using $\hat{a}=F^{-1}\left(1-\frac{c}{\lambda}\right)$ leads to

$$
\lambda \int_{\bar{a}}^{\hat{a}} \frac{V_{t}(a)}{V_{t}(\bar{a})[1-F(\bar{a})]} d F(a)=\lambda \int_{\bar{a}}^{\hat{a}} \frac{\operatorname{Pr}\left(a_{T}^{-i}<a\right)}{\operatorname{Pr}\left(a_{T}^{-i}<\bar{a}\right)[1-F(\bar{a})]} d F(a)=1 .
$$

The equilibrium strategies enable us to express the above equation explicitly as

$$
\lambda \int_{\bar{a}}^{\hat{a}} \frac{e^{-\lambda T(1-F(\bar{a}))}+\left[1-e^{-\lambda T(1-F(\bar{a}))}\right] \frac{F(a)-F(\bar{a})}{1-F(\bar{a})}}{e^{-\lambda T(1-F(\bar{a}))}[1-F(\bar{a})]} d F(a)=1 .
$$

Notice that the cutoff $\bar{a}$ in the equilibrium with strategic delay, while depends on $T$, does not depend on $t$, and is constant throughout the contest.

Proposition 9 formally presents the symmetric equilibrium with strategic delay:

Proposition 9 (Symmetric Equilibrium with Strategic Delay) In a two-agent public contest with deadline $T<\infty$ and 0 opportunity cost of staying in the contest without searching, there is a symmetric equilibrium with strategic delay. Specifically, denoting agent $i$ 's best innovation at $t$ as $a_{t}$,

1. when there is no previous submission, agent $i$ searches at $t<T$ if $a_{t}<\bar{a}$ ( $\bar{a}$ defined in (14)), submits $a_{t}$ if $a_{t} \geq \hat{a}$, and if $a_{t} \in[\bar{a}, \hat{a})$, stops searching without submitting until time $T$ or until a submission from the opponent at a time before $T$; 
2. when the opponent $j$ submits $a_{j}$ before $T$, agent $i$ stops searching and submits his best innovation immediately if $a_{j} \geq \hat{a}$, and agent $i$ continues to search with cutoff $a_{j}$ otherwise.

In Proposition 9, strategic delay, where the agents stop searching and waiting in the contest, occurs with positive probability on the equilibrium path, and the agents behave as if they were coordinating on a cutoff lower than $\hat{a}$. As mentioned, strategic delay arises in equilibrium due to each agent's unwillingness to reveal his best innovation below $\hat{a}$, which once revealed may induce the opponent to search further, leading to suboptimal expected outcomes to the agent.

Consider next the hidden policy. Notice that regardless of whether the opportunity cost of remaining in the contest is zero or positive, there is a unique symmetric equilibrium outcome where each agent searches with the cutoff $a^{*}$, which is outcome equivalent to the symmetric equilibrium in Proposition $1 .{ }^{39}$ It is then natural to again compare equilibrium behavior in the public contest and the hidden contest in this setting. We first present some numerical results on the equilibrium cutoffs:
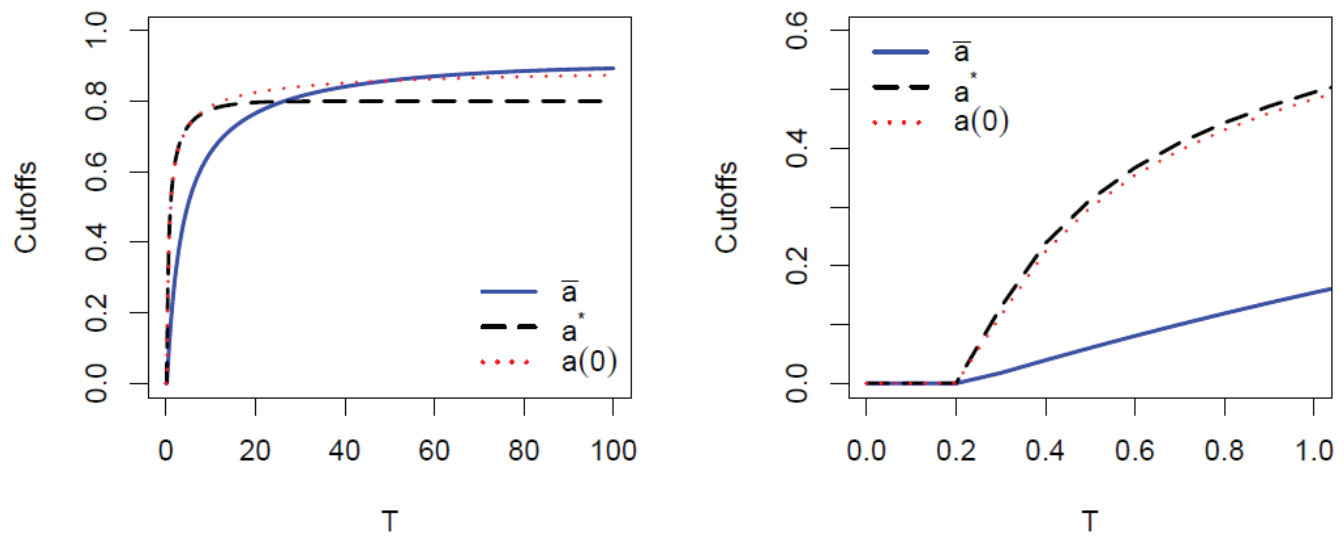

Figure 4. Comparing Equilibrium Cutoffs $\bar{a}, a^{*}$, and $\alpha_{0}^{*}$ in Propositions 9, 1, and 2. Parameters: $\lambda=1, c=0.1$, and $F$ being Uniform.

Figure 4 compares the equilibrium cutoffs $\bar{a}$ (blue solid curve), $a^{*}$ (black dashed curve) in the hidden contest, and $\alpha_{0}^{*}$ in the two-agent public contest (red dotted curve, labeled as $a(0))$ as functions of the contest deadline $T .{ }^{40}$ The left panel shows the cutoffs when $T$

\footnotetext{
${ }^{39}$ In a symmetric equilibrium in the hidden contest with no opportunity cost of time, an agent can wait until $T$ or submit his best innovation immediately after passing $a^{*}$. In every symmetric equilibrium, all agents however start searching immediately at $t=0$ without delay, since the winning cutoff $a^{*}$ is endogeneously determined by $T$.

${ }^{40}$ Recall that the equilibrium cutoff $\alpha_{0}^{*}$ is defined in (5) by setting $t=0$. In particular, it is the beginning equilibrium cutoff in the public contest when $c$ is both the opportunity and fiduciary cost.
} 
varies from 0 to 100, and the right panel shows the plot of the left panel by zooming the proportion when $T \in[0,1]$. First, notice that all three cutoffs are strictly increasing in the deadline $T$. Second, the agents search less intensively in the public contest when $T$ is small, i.e., $\alpha_{0}^{*}<a^{*}$ and $\bar{a}<a^{*}$. This is qualitatively similar to that in Figure 1 , where we compare $a^{*}$ and $\alpha_{t}^{*}$ when there is no strategic delay. Compared to $\alpha_{0}^{*}$, each agent, knowing that the opponent will most likely hold his best innovation without submitting, settles on an even lower cutoff $\bar{a}$ than $\alpha_{0}^{*}$. Finally, when $T$ is relatively large, we have $\alpha_{0}^{*}>a^{*}$ and $\bar{a}>a^{*}$, i.e., the agents search with higher cutoffs in the public contest than in the public contest, with or without strategic delay.

Finally, notice that we have (recall (6) in Section 3.2 and use (14)):

$$
\lim _{T \rightarrow \infty} \alpha_{t}^{*}=\lim _{T \rightarrow \infty} \bar{a}=\hat{a}
$$

Hence, both agents will search with the time-independent cutoff $\hat{a}$ in equilibrium in a public contest with an infinite horizon, whether or not there is a positive cost of staying in the contest without searching. This is intuitive: In an infinite-horizon public contest, an opponent will eventually obtain an innovation above $\hat{a}$ and holding the best innovation (below $\hat{a}$ ) without searching only increases the probability of being preempted by the opponent. Hence, each agent searches without delay until he reaches $\hat{a}$. Given this result, the ranking between the public policy and the hidden policy stays the same for the sponsor in this alternative setting:

Corollary 2 In a two-agent contest with an infinite horizon and 0 opportunity cost of staying in the contest without searching, the sponsor again strictly prefers the public policy to the hidden policy.

In particular, Corollary 2 implies that our ranking result between the public policy and the hidden policy remains the same, even if we allow the agents to wait in the contest without incurring any opportunity cost.

\section{Conclusion}

We have analyzed various information disclosure policies in a classic dynamic research contest framework, where the contest sponsor chooses how to strategically disclose the competing agents' final submissions over time, so as to influence the agents' innovation incentives to her own benefit. We have imposed arguably minimal information requirement for the sponsor, who does not observe the agents' search behavior or outcomes and 
only employs information on final submissions as instruments, which is generated dynamically and endogenously in the contest. Our analysis has uncovered various incentives and economic forces that different disclosure policies can bring about. In particular, we have identified the public disclosure policy as a best policy, and the hidden policy as a worst policy, within a natural class of disclosure policies. Overall, the analysis in the paper implies that information disclosure policies can be a surprisingly effective tool, which can be potentially useful for real-world dynamic contests.

\section{Appendix: Omitted Proofs.}

\section{Proof of Proposition 1.}

In a symmetric equilibrium, each agent chooses an identical cutoff $a^{*}$. Recall the instantaneous gain $\Delta \Pi\left(a, a^{*}\right)$ from searching at state $a$ given in (2). In equilibrium, an agent with the cutoff state $a^{*}$ is indifferent between searching and not searching, i.e., $\Delta \Pi\left(a^{*}, a^{*}\right)=0$, or using $(1)$

$$
\begin{aligned}
& \lambda \int_{a^{*}}^{1}\left[1-e^{-\lambda T\left(1-F\left(a^{*}\right)\right)}\right] \frac{F(x)-F\left(a^{*}\right)}{1-F\left(a^{*}\right)} d F(x)=c \\
\Leftrightarrow & \frac{\lambda}{2}\left[1-F\left(a^{*}\right)\right]\left[1-e^{-\lambda T\left(1-F\left(a^{*}\right)\right)}\right]=c .
\end{aligned}
$$

In addition, given $\Delta \Pi\left(a^{*}, a^{*}\right)=0$, it can be verified that

$$
\Delta \Pi\left(a, a^{*}\right)=\left\{\begin{array}{l}
\lambda\left[1-e^{-\lambda T\left(1-F\left(a^{*}\right)\right)}\right] \int_{a}^{1}[F(x)-F(a)] d F(x)-c<0 \text { for } a>a^{*} \\
\lambda \int_{a}^{a^{*}}\left[e^{-\lambda T(1-F(x))}-e^{-\lambda T(1-F(a))}\right] d F(x)>0 \text { for } a<a^{*}
\end{array}\right.
$$

Hence, $a^{*}$ is unique and an $a^{*}$-cutoff strategy is $i$ 's best response when $j$ plays the $a^{*}$-cutoff strategy. In addition, we evaluate the two end points $a^{*}=0$ and $a^{*}=1$ to obtain that $a^{*} \in(0,1)$ if $0<c<\lambda\left(1-e^{-\lambda T}\right) / 2$.

Finally, consider the possibility where both agents search with cutoff $a^{*}=0$. Given $j$ 's cutoff $a^{*}=0, i$ 's instantaneous gain from searching starting from $a=0$ is

$$
\Delta \Pi\left(a=0, a^{*}=0\right)=\lambda \int_{0}^{1} Z(x \mid 0, T) d F(x)-c=\frac{1+e^{-\lambda T}}{2}-\frac{c}{\lambda}>0 .
$$

If in addition, $c \in\left[\frac{1-e^{-\lambda T}}{2}, \frac{\lambda}{2}\right)$, then the agent has strict incentives to search at state $a=0$, but will stop searching after obtaining a single draw above 0, i.e., the agent searches with cutoff $a^{*}=0$. 


\section{Proof of Proposition 2.}

The agents' incentives to search with a cutoff $\alpha_{t}^{*}$ when no submission has been made and after a submission from the opponent have already been discussed in the text. Here we verify that the cutoff value $\alpha_{t}^{*}$ is uniquely determined in the indifference condition (4), leading to $\alpha_{t}^{*} \in[0, \hat{a})$, and investigate the agents' incentives to search at state $a_{t}=0$.

We first use (3) to simplify the indifference condition (4) to be

$$
\begin{gathered}
\int_{\alpha_{t}^{*}}^{\hat{a}} e^{-\lambda(T-t)[1-F(a)]} d F(a)=\left[1-F\left(\alpha_{t}^{*}\right)\right] e^{-\lambda(T-t)\left[1-F\left(\alpha_{t}^{*}\right)\right]} \\
\Leftrightarrow \frac{e^{\left[\lambda\left(1-F\left(\alpha_{t}^{*}\right)\right)-c\right](T-t)}-1}{T-t}=\lambda\left[1-F\left(\alpha_{t}^{*}\right)\right] .
\end{gathered}
$$

Recall that an agent's instantaneous gain from searching at state $a_{t}$ is

$$
\Delta \Pi\left(a_{t}\right)=\lambda \int_{a_{t}}^{1}\left[V(a)-V\left(a_{t}\right)\right] d F(a)-c .
$$

The instantaneous gain for an agent to search at state $\hat{a}$ is

$$
\Delta \Pi(\hat{a})=-\lambda[1-F(\hat{a})] V(\hat{a})=-c .
$$

This is intuitive since searching at innovation $\hat{a}$ only incurs a net loss of $c$. This implies that $\alpha_{t}^{*}<\hat{a}$. Notice that this calculation also implies that for any fixed $T$, the instantaneous gain for states less than but close to $\hat{a}$ is also strictly negative - so $\hat{a}$ cannot be an equilibrium cutoff for the finite public contest game.

Now consider an agent's incentives for $a_{t} \in[0, \hat{a})$. Since $V\left(a_{t}\right)$ is strictly increasing in $a_{t}$, we have that $\Delta \Pi\left(a_{t}\right)$ is strictly decreasing in $a_{t}$. We discuss two cases. First, if $a_{t} \in(0, \hat{a}), \Delta \Pi\left(a_{t}\right)>0$ implies that (15) has a unique $\alpha_{t}^{*}>a_{t}$ such that $\Delta \Pi\left(\alpha_{t}^{*}\right)=0$, and an agent continues to search. If $\Delta \Pi\left(a_{t}\right) \leq 0$, we have $a_{t}>\alpha_{t}^{*}=0$ and the agent stops searching. Second, if $a_{t}=0$, the instantaneous gain from search is (recall that innovation 0 is useless and hence $V(0)=0)$

$$
\begin{aligned}
\Delta \Pi(0) & =\lambda(1-F(\hat{a}))+\lambda \int_{0}^{\hat{a}} V(a) d F(a)-\lambda V(0)-c \\
& =\lambda \int_{0}^{\hat{a}} e^{-\lambda(T-t)[1-F(a)]} d F(a)>0 .
\end{aligned}
$$

An agent with $a_{t}$ hence always has strict incentives to search at any $t$. If there is a solution $\alpha_{t}^{*} \in(0, \hat{a})$ to $(15)$, the agent searches with such a cutoff $\alpha_{t}^{*}$. Consider the remaining possibility that at some $t,(15)$ admits no solution in $(0, \hat{a})$, i.e., $\Delta \Pi(a)<0$ 
for all $a \in(0, \hat{a})$. While the agent still searches in the instant as $\Delta \Pi(0)>0$, the agent stops immediately after drawing a non-zero innovation, say $\beta$, since now we necessarily have $\Delta \Pi(\beta)<0$, i.e., the agent stops once obtaining $\beta$. Hence, the agent in this scenario searches with cutoff $\alpha_{t}^{*}=0$.

\section{Proof of Proposition 3.}

To prove Proposition 3, we need to verify the agents' incentives in the equilibrium and to show the existence of a unique (and well-defined) equilibrium cutoff $a^{*}$. First, recall $\Delta \Pi\left(a, a^{*}\right)$ defined in $(7)$ and calculate

$$
\begin{aligned}
& \frac{d \Delta \Pi\left(a, a^{*}\right)}{d a}=-\lambda \int_{a}^{1}(N-1) Z^{N-2}\left(a \mid a^{*}, T\right) \frac{d Z\left(a \mid a^{*}, T\right)}{d a} d F(x) \\
& \text { where } \frac{d Z\left(a \mid a^{*}, T\right)}{d a}=\left\{\begin{array}{l}
e^{-\lambda T[1-F(a)]} \lambda T f(a), \text { if } a \in\left[0, a^{*}\right) \\
{\left[1-e^{-\lambda T\left[1-F\left(a^{*}\right)\right]}\right] \frac{f(a)}{1-F\left(a^{*}\right)}, \text { if } a \in\left[a^{*}, 1\right)}
\end{array}\right.
\end{aligned}
$$

Hence, the instantaneous gain $\Delta \Pi\left(a, a^{*}\right)$ strictly decreases in $a$, implying that $\Delta \Pi\left(a, a^{*}\right)=$ 0 occurs at most once. This is intuitive since it makes less sense to search if an agent's best innovation is higher. By the definition of $a^{*}$, i.e., $\Delta \Pi\left(a^{*}, a^{*}\right)=0$, each agent has strict incentives to search below $a^{*}$ and strict incentives not to search above $a^{*}$.

We now show that $a^{*}$ is well-defined, i.e., $a^{*} \in[0,1)$. It suffices to evaluate

$$
\begin{aligned}
\lim _{a^{*} \downarrow 0} \Delta \Pi\left(a^{*}, a^{*}\right) & =\lambda \int_{0}^{1}\left\{\left[e^{-\lambda T}+\left(1-e^{-\lambda T}\right) F(x)\right]^{N-1}-e^{-\lambda T(N-1)}\right\} d F(x)-c \\
& =\frac{\lambda}{N} \frac{1+(N-1) e^{-\lambda T N}-N e^{-\lambda T(N-1)}}{\left(1-e^{-\lambda T}\right)}-c \\
& =\frac{\lambda}{N} \sum_{k=0}^{N-1}\left(e^{-k \lambda T}-e^{-(N-1) \lambda T}\right)-c \\
& \equiv c^{*}-c(\text { recall }(9)) .
\end{aligned}
$$

If $\lim _{a^{*} \rightarrow 0} \Delta \Pi\left(a^{*}, a^{*}\right)>0$, then we have a unique and interior equilibrium cutoff value $a^{*} \in(0,1)$. It is easy to verify that $\lim _{T \rightarrow 0} c^{*}=0$ and $\lim _{T \rightarrow \infty} c^{*}=\frac{\lambda}{N}$. For $N=2$, we have that $c^{*}<\frac{\lambda}{N}$ for all $T$, while if $N \geq 3$, it is however possible that $c^{*}>\frac{\lambda}{N}$. Hence, if $c^{*}>c$, then all agents search with an interior cutoff $a^{*} \in(0,1)$, while if $c^{*} \leq c$ (impossible if $\left.c^{*}>\frac{\lambda}{N}\right)$, the condition $\Delta \Pi\left(a^{*}, a^{*}\right)=0$ does not admit any solution $a^{*} \in[0,1)$. In this case, we impose $a^{*}=0$ and show below that each agent still has positive incentive to 
search at state $a=0$. Indeed, we can calculate that

$$
\begin{aligned}
\Delta \Pi\left(0, a^{*}=0\right) & =\lambda \int_{0}^{1}\left[e^{-\lambda T}+\left(1-e^{-\lambda T}\right) F(x)\right]^{N-1} d F(x)-c \\
& =\frac{\lambda\left(1-e^{-\lambda T N}\right)}{N\left(1-e^{-\lambda T}\right)}-c>0 .
\end{aligned}
$$

However, once an agent obtains a state above 0, the agent will stop due to our above analysis on $\frac{d \Delta \Pi\left(a, a^{*}\right)}{d a}$. Each agent hence searches with the minimum cutoff $a^{*}=0$.

\section{Proof of Lemma 1.}

Consider the $(N-K)$-auxiliary contest and a history of no previous submissions. Define an agent's expected payoff from stopping at a state $a_{t} \in[0, \hat{a})$ at time $t<T$ as

$$
V\left(a_{t}\right)=\left\{\begin{array}{l}
1, \text { if } a_{t} \geq \hat{a} \\
\mu_{t} \cdot e^{-\lambda(T-t)\left[1-F\left(a_{t}\right)\right](N-K-1)}, \text { if } a_{t} \in(0, \hat{a}) \\
0, \text { if } a_{t}=0
\end{array}\right.
$$

where $\mu_{t}$ again is the agent's belief that his opponents' current states are all below $a_{t}$ given the agent's (private) history at $t$, and $\mu_{t} \geq e^{-\lambda(N-K-1)}>0$ for $a_{t} \in(0, \hat{a})$. The instantaneous gain from searching and stopping for the agent is

$$
\Delta \Pi\left(a_{t}\right)=\lambda \int_{a_{t}}^{1}\left[V(a)-V\left(a_{t}\right)\right] d F(a)-c .
$$

Notice that $V\left(a_{t}\right)$ is strictly increasing in $a_{t}$ while $\Delta \Pi\left(a_{t}\right)$ is strictly decreasing in $a_{t}$. And the equilibrium cutoff $\alpha_{t}^{*}(K)$ is implicitly defined by $\Delta \Pi\left(\alpha_{t}^{*}(K)\right)=0$, i.e.,

$$
\lambda \int_{\alpha_{t}^{*}(K)}^{\hat{a}} V(a) d F(a)=\lambda\left[1-F\left(\alpha_{t}^{*}(K)\right)\right] V\left(\alpha_{t}^{*}(K)\right)
$$

or equivalently,

$$
\frac{e^{\lambda(T-t)(N-K-1)\left[F(\hat{a})-F\left(\alpha_{t}^{*}(K)\right)\right]}-1}{(T-t)(N-K-1)}=\lambda\left[1-F\left(\alpha_{t}^{*}(K)\right)\right]
$$

which is a generalization of (5) in the two-player case. The above characterizes an interior cutoff $\alpha_{t}^{*}(K) \in(0, \hat{a})$, while the 'corner' solution $\alpha_{t}^{*}(K)=0$ arises if

$$
\frac{e^{\lambda(T-t)(N-K-1) F(\hat{a})}-1}{(T-t)(N-K-1)} \leq \lambda
$$


Finally, the comparative statics $\frac{d \alpha_{t}^{*}(K)}{d t}<0, \frac{d \alpha_{t}^{*}(K)}{d K}<0, \frac{d \alpha_{0}^{*}(K)}{d T}>0$ can all be immediately verified by implicit differentiations. To calculate $\lim _{T \rightarrow \infty} \alpha_{0}^{*}(K)$, rewrite (16) to be

$$
\lambda(N-K-1)\left[F(\hat{a})-F\left(\alpha_{t}^{*}(K)\right)\right]=\frac{\ln \left\{1+\lambda(N-K-1)(T-t)\left[1-F\left(\alpha_{t}^{*}(K)\right)\right]\right\}}{T-t} .
$$

An implicit differentiation on $T$ leads to (recall that $F$ is continuously differentiable and has bounded density)

$$
\lim _{T \rightarrow \infty} \frac{d \alpha_{t}^{*}(K)}{d T}=0 .
$$

An application of the l'Hopital's rule implies $\lim _{T \rightarrow \infty} \alpha_{t}^{*}(K)=\hat{a}=F^{-1}\left(1-\frac{c}{\lambda}\right)$.

\section{Proof of Proposition 4.}

Part 1 and part 2 of the proposition are straightforward. We focus on part 3 hereafter.

Consider a history with exactly $K$ submissions, $K \in\{0, \ldots, N-2\}$ and the highest submission is $a(K)<\hat{a}$. We first show that each remaining agent $i$ has strictly positive incentive to search at instant $t$. Let agent $i$ 's state at $t$ be $a_{i}$. Then agent $i$ 's expected payoff from stopping and submitting $a_{i}$ is

$$
V\left(a_{i}\right)=\left\{\begin{array}{l}
1, \text { if } a_{i} \geq \hat{a} \\
\mu_{t} \cdot e^{-\lambda(T-t)\left[1-F\left(a_{i}\right)\right](N-K-1)}, \text { if } a_{i} \in(a(K), \hat{a}) \\
0, \text { if } a_{i}<a(K)
\end{array}\right.
$$

where as before $\mu_{t}>0$ is agent $i$ 's belief that the $(N-K-1)$ other remaining agents' states are below $a(K)$. Suppose $a_{i}<a(K)$. Then agent $i$ 's instantaneous gain from searching and then submitting at instant $t$ is (notice that $V\left(a_{i}\right)=0$ here):

$$
\lambda \int_{a(K)}^{1} V(a) d F(a)-c=\lambda \int_{a(K)}^{\hat{a}} \mu_{t} \cdot e^{-\lambda(T-t)\left[1-F\left(a_{i}\right)\right](N-K-1)} d F(a)>0,
$$

i.e., agent $i$ will continue to search if $a_{i}<a(K)$ regardless of his belief $\mu_{t}$.

Suppose $a(K)<\alpha_{t}^{*}(K)$, where recall that $\alpha_{t}^{*}(K)$ is defined in Lemma 1 . Then agent $i$ will be indifferent between searching and not searching at some $a^{*}$ such that

$$
\lambda \int_{a^{*}}^{\hat{a}} e^{-\lambda(T-t)[1-F(a)](N-K-1)} d F(a)=\lambda\left[1-F\left(a^{*}\right)\right] e^{-\lambda(T-t)\left[1-F\left(a^{*}\right)\right](N-K-1)}
$$

which is exactly the definition of $\alpha_{t}^{*}(K)$, or $a^{*}=\alpha_{t}^{*}(K)$. Hence, in this case, the remaining agents search with cutoff $\alpha_{t}^{*}(K)$ at instant $t$. Notice that this is also consistent with (17).

Now suppose $a(K) \in\left(\alpha_{t}^{*}(K), \hat{a}\right)$. Our above analysis in (17) shows that a remaining 
agent $i$ with a best innovation $a_{i}<a(K)$ will continue to search. For a remaining agent $i$ with a best innovation $a_{i}>a(K)$, the instantaneous gain from searching for instant $t$ is (notice that $V\left(a_{i}\right)>0$ here):

$$
\Delta \Pi\left(a_{i}\right)=\lambda \int_{a_{i}}^{1}\left[V(a)-V\left(a_{i}\right)\right] d F(a)-c<0,
$$

since (1) $V\left(a_{i}\right)$ is strictly increasing in $a_{i}$ and hence $\Delta \Pi\left(a_{i}\right)$ is strictly decreasing in $a_{i}$ and $(2) a_{i}>a(K)>\alpha_{t}^{*}(K)$. Hence, agent $i$ searches with $a(K)$ as the relevant cutoff. ${ }^{41}$

Summarizing, after $K \in\{0, \ldots, N-2\}$ submissions and the best submission being $a(K)$, each remaining agent searches with cutoff $\max \left\{a(K), \alpha_{t}^{*}(K)\right\}$.

\section{Proof of Proposition 6.}

We first verify the specified behavior is an equilibrium, by considering each agent's incentives before and after the sponsor's announcement.

First, suppose the sponsor announces at some $\hat{t}>0$ that there are already $k$ submissions, each passing the cutoff $a^{k}=F^{-1}\left(1-\frac{k c}{\lambda}\right)$. Consider an agent who has not submitted at or before $\hat{t}$. The agent's instantaneous gain from searching at $t>\hat{t}$ with his best innovation at $t$ being $a^{\prime} \leq a^{k}$ is (recall that if the agent submits $a^{\prime}$, he will get 0 with probability one given the sponsor's announcement):

$$
\Delta \Pi^{k}\left(a^{\prime}\right)=\lambda \int_{a^{k}}^{1}\left[\frac{F(a)-F\left(a^{k}\right)}{1-F\left(a^{k}\right)}\right]^{k} d F(a)-c=\frac{\lambda\left[1-F\left(a^{k}\right)\right]}{k+1}-c=\frac{k c}{k+1}-c<0 .
$$

If however the agent's best innovation at $t$ being $a^{\prime}>a^{k}$, the agent's instantaneous gain from searching at $t>\hat{t}$ is

$$
\begin{aligned}
\Delta \Pi^{k}\left(a^{\prime}\right) & =\lambda\left[1-F\left(a^{\prime}\right)\right] \int_{a^{\prime}}^{1}\left[\frac{F(a)-F\left(a^{k}\right)}{1-F\left(a^{k}\right)}\right]^{k} d F(a)-\lambda\left[1-F\left(a^{\prime}\right)\right]\left[\frac{F\left(a^{\prime}\right)-F\left(a^{k}\right)}{1-F\left(a^{k}\right)}\right]^{k}-c \\
& <\frac{\lambda\left[1-F\left(a^{\prime}\right)\right]\left[1-F\left(a^{k}\right)\right]}{k+1}-c<0,
\end{aligned}
$$

which is intuitive since by submitting $a^{\prime}$, the agent can already win the prize with positive probability, and the instantaneous gain from searching more at $a^{\prime}>a^{k}$ is thus smaller. Hence, we conclude that upon receiving the sponsor's announcement at $\hat{t}$, every remaining

\footnotetext{
${ }^{41}$ Notice that the derivation of $a(K)$ being the cutoff here is different from our "usual" approach in deriving a cutoff value, since there is a discontinuous upward jump in an agent's expected payoff from a state below $a(K)$ to a state above $a(K)$.
} 
$(N-k)$ agent has no incentive to search regardless of the agent's best innovation.

Next, consider a time $t$ when the sponsor has not made any announcement. An agent's instantaneous gain from searching at any time point depends on his belief of how many other agents have obtained innovations above $a^{k}$ already. Suppose an agent's best innovation at $t$ is $a^{\prime} \leq a^{k}$. Regardless of this agent's belief of how many other agents have already submitted their innovations above $a^{k}$ (which is at least 0 and at most $(k-1)$ agents), the agent's instantaneous gain from searching at $t$ is

$$
\Delta \hat{\Pi}^{k}\left(a^{\prime}\right)=\lambda \int_{a^{k}}^{1}\left[\frac{F(a)-F\left(a^{k}\right)}{1-F\left(a^{k}\right)}\right]^{k-1} d F(a)-c=\frac{\lambda\left[1-F\left(a^{k}\right)\right]}{k}-c=0 .
$$

The reason is that given an infinite horizon and the agents' equilibrium behavior (which is symmetric and in pure strategies), there will ultimately be $k$ submissions with innovations all above $a^{k}$. In addition, since there are strictly less than $k$ submissions at $t$ and the probability of there being $k$ submissions in the next instant $(t+\Delta t)$ is negligible, the agent can guarantee to be one of the $k$ "winning" agents if he obtains an innovation above $a^{k}$ and submits it in the next instant. Given (18), each agent hence has (weak) incentives to search in the next instant if his best innovation is currently less than $a^{k}$.

Again consider a time $t$ when there has not been any announcement. Suppose an agent's best innovation at $t$ is $a^{\prime}>a^{k}$. The agent's instantaneous gain is:

$$
\begin{aligned}
& \Delta \hat{\Pi}^{k}\left(a^{\prime}\right) \\
= & \lambda\left[1-F\left(a^{\prime}\right)\right] \int_{a^{\prime}}^{1}\left[\frac{F(a)-F\left(a^{k}\right)}{1-F\left(a^{k}\right)}\right]^{k-1} d F(a)-\lambda\left[1-F\left(a^{\prime}\right)\right]\left[\frac{F\left(a^{\prime}\right)-F\left(a^{k}\right)}{1-F\left(a^{k}\right)}\right]^{k-1}-c \\
< & \lambda\left[1-F\left(a^{\prime}\right)\right] \int_{a^{\prime}}^{1}\left[\frac{F(a)-F\left(a^{k}\right)}{1-F\left(a^{k}\right)}\right]^{k-1} d F(a)-c \\
= & \frac{\lambda\left[1-F\left(a^{\prime}\right)\right]\left[1-F\left(a^{k}\right)\right]}{k}-c<0 .
\end{aligned}
$$

Hence, after a history of no announcement, an agent with a best innovation above $a^{k}$ has no incentive to search further and submits his best innovation immediately. We conclude that the specified behavior for the agents constitutes an equilibrium.

Finally, equilibrium uniqueness follows from the definition of the simple $k$-disclosure policy, and that we focus on symmetric equilibrium in pure strategies: Though each agent is indifferent between searching and not on the path of play, a standard argument shows that there cannot be an equilibrium where no one searches in the contest. 


\section{Proof of Proposition 7.}

Recall that an innovation with state $a$ is worth $v(a)$ to the sponsor and $v(\cdot)$ is strictly increasing. It suffices to show that for $k \in\{1, \ldots, N-1\}$, the distribution of the winning innovation in a symmetric equilibrium outcome with $k$ effective submissions first-order stochastically dominates that of the winning innovation in a symmetric equilibrium with $(k+1)$ effective submissions.

We denote the distribution of the winning innovation in a symmetric equilibrium with $\ell$ effective submissions as $G^{\ell}, \ell \in\{1, \ldots, N\}$. Since exactly $\ell$ agents obtain innovations at least $a^{\ell}$ in a symmetric equilibrium outcome with $\ell$ effective submissions, we have:

$$
G^{\ell}(a)=\left[\frac{F(a)-F\left(a^{\ell}\right)}{1-F\left(a^{\ell}\right)}\right]^{\ell} \text {, if } a \in\left[a^{\ell}, 1\right] ; G^{\ell}(a)=0 \text { otherwise. }
$$

In addition, given that $a^{\ell}=F^{-1}\left(1-\frac{\ell c}{\lambda}\right)$, we have $a^{k}>a^{k+1}$ for $k \in\{1, \ldots, N-1\}$. We need to show that $G^{k}(a)$ first-order stochastically dominates $G^{k+1}(a)$.

For $a \in\left[a^{k+1}, a^{k}\right]$, we have $G^{k+1}(a)>G^{k}(a)=0$. For $a \in\left(a^{k}, 1\right]$, notice that $G^{k+1}(1)=G^{k}(a)=1$. For $a \in\left(a^{k}, 1\right)$, we have

$$
\begin{aligned}
\frac{G^{k+1}(a)}{G^{k}(a)} & =\frac{\left[F(a)-F\left(a^{k+1}\right)\right]^{k+1}}{\left[F(a)-F\left(a^{k}\right)\right]^{k}} \frac{\left[1-F\left(a^{k}\right)\right]^{k}}{\left[1-F\left(a^{k+1}\right)\right]^{k+1}} \\
& =\frac{\left[F(a)-F\left(a^{k+1}\right)\right]^{k+1}}{\left[F(a)-F\left(a^{k}\right)\right]^{k}} \frac{\lambda k^{k}}{c(k+1)^{k+1}} .
\end{aligned}
$$

For $x:=F(a) \in\left(F\left(a^{k}\right), 1\right)$, define

$$
H(x)=\frac{\left[x-F\left(a^{k+1}\right)\right]^{k+1}}{\left[x-F\left(a^{k}\right)\right]^{k}} .
$$

It can be verified that

$$
\frac{d H^{2}(x)}{d x^{2}}=k(k+1)\left[\frac{\left[x-F\left(a^{k+1}\right)\right]^{k-1}}{\left[x-F\left(a^{k}\right)\right]^{k}}-2 \frac{\left[x-F\left(a^{k+1}\right)\right]^{k}}{\left[x-F\left(a^{k}\right)\right]^{k+1}}+\frac{\left[x-F\left(a^{k+1}\right)\right]^{k+1}}{\left[x-F\left(a^{k}\right)\right]^{k-2}}\right]>0
$$

where the last inequality is a result of a standard two-variable Arithmetic Geometric Means (AGM) inequality. Hence, $H(x)$ is strictly convex in $x .^{42}$

\footnotetext{
${ }^{42}$ Notice that the same thing holds if we consider $H(\cdot)$ as a function of $a$, due to the fact that $F(\cdot)$ is a differentiable and strictly increasing function of $a$.
} 
Hence, $H(x)$ achieves its minimum at its unique stationary point $x^{*}$ :

$$
\frac{d H(x)}{d x}=0 \Longrightarrow \frac{k+1}{k}=\frac{x^{*}-F\left(a^{k+1}\right)}{x^{*}-F\left(a^{k}\right)} \Longrightarrow x^{*}=1 .
$$

This further implies that

$\frac{G^{k+1}(a)}{G^{k}(a)}>\frac{\left[1-F\left(a^{k+1}\right)\right]^{k+1}}{\left[1-F\left(a^{k}\right)\right]^{k}} \frac{\lambda k^{k}}{c(k+1)^{k+1}}=1$ for $a \in\left(a^{k}, 1\right)$ and $\frac{G^{k+1}(a)}{G^{k}(a)}=1$ for $a=1$

We conclude that $G^{k}(a)$ (strictly) first-order stochastically dominates $G^{k+1}(a)$, and the sponsor strictly prefers $G^{k}(a)$ to $G^{k+1}(a)$.

\section{Proof of Proposition 9.}

We have shown in the text that there is a unique, well-defined cutoff $\bar{a} \in[0, \hat{a})$ for (14), and we have also verified the agents' incentives to search with cutoff $\bar{a}$ after a history with no submission. In addition, an agent's incentive to search or stop after a submission from the opponent (either below $\hat{a}$ or above $\hat{a}$ ) is similar to that in Proposition 2. In the remainder, we verify the agents' incentives after other histories.

First, consider an agent with innovation $a \in[\bar{a}, \hat{a})$ at $t<T$. The agent only wins when the opponent submits an innovation above $a$. A submission of innovation $a$ by the agent prompts the opponent to search with cutoff $a$ (in the event that the opponent's current state is below $a$ ), while the opponent only searches with the strictly lower cutoff $\bar{a}$ if the agent holds $a$ until $T$. Hence, intuitively submitting $a$ at $t$ is strictly worse than holding $a$ until $T$. To verify this explicitly, the agent's continuation payoff if he holds his innovation $a$ without submitting until $T$ is (recall that the opponent uses $\bar{a}$ as the cutoff in his search and $Z(a \mid \bar{a}, T)$ is defined in (1)):

$$
V_{t}(a)=e^{-\lambda T[1-F(\bar{a})]}+\left(1-e^{-\lambda T[1-F(\bar{a})]}\right) \frac{F(a)-F(\bar{a})}{1-F(\bar{a})}=Z(a \mid \bar{a}, T)
$$

while the agent's continuation payoff from submitting $a$ immediately at $t$ is

$$
V_{t}^{S}(a)=Z(a \mid \bar{a}, T) e^{-\lambda(T-t)[1-F(a)]}<V_{t}(a)
$$

Hence submitting innovation $a$ at $T$ is strictly better than submitting $a$ at $t$.

Second, consider an agent with innovation $a \in[0, \bar{a})$. Observe that submitting $a$ immediately is at most as good as stopping searching and holding a until $T$, since holding 
$a$ without searching does not incur any cost and also allows for a continual search (if necessary) at a later point. On the other hand, our discussion on equation (13) in the text shows that searching given current innovation $a<\bar{a}$ is strictly better than stopping and holding $a$. This implies that given innovation $a \in[0, \bar{a})$, searching is strictly better than submitting $a$.

\section{References}

[1] Aoyagi, M. (2010), "Information Feedback in a Dynamic Tournament," Games and Economic Behavior, 70: 242-260.

[2] Burton, M.D. and T. Nicholas (2017), "Prizes, patents and the search for longitude," Explorations in Economic History, 64: 21-36.

[3] Che, Y-K., and I. Gale (2003), "Optimal Design of Research Contests," American Economic Review, 93: 646-671.

[4] Che, Y-K., and J. Hörner (2018), "Recommender Systems as Mechanisms for Social Learning," Quarterly Journal of Economics, 133: 871-925.

[5] Corchón, L.C. (2007), "The Theory of Contests: A Survey," Review of Economic Design, 11: 69-100.

[6] Daley, B. and R. Wang (2018), "When to Release Feedback in a Dynamic Tournament," Decision Analysis, 15: 11-26.

[7] Ederer, F. (2010), "Feedback and Motivation in Dynamic Tournaments," Journal of Economics \& Management Strategy, 19: 733-769.

[8] Ely, J.C. (2017), "Beeps," American Economic Review, 107, 31-35.

[9] Ely, J.C., and M. Szydlowski (2017), "Moving the Goalposts," Journal of Political Economy, forthcoming.

[10] Fullerton, R.L., and P.R. McAfee (1999), "Auctioning Entry into Tournaments," Journal of Political Economy, 107: 573-605.

[11] Gill, D. (2008), "Strategic Disclosure of Intermediate Research Results," Journal of Economics 8 Management Strategy, 17: 733-758. 
[12] Goltsman, M. and A. Mukherjee (2011), "Interim Performance Feedback in Multistage Tournaments: The Optimality of Partial Disclosure," Journal of Labor Economics, 29: 229-265.

[13] Halac, M., N. Kartik, and Q. Liu (2017), "Contests for Experimentation," Journal of Political Economy, 125: 1523-1569.

[14] Konrad, K.A. (2009), Strategy and Dynamics in Contests, Oxford University Press.

[15] Lang, M., C. Seel, and P. Strack (2014), "Deadlines in Stochastic Contests," Journal of Mathematical Economics, 52: 134-142.

[16] Moldovanu, B., and A. Sela (2001), "The Optimal Allocation of Prizes in Contests," American Economic Review, 91: 542-558.

[17] Moscarini, G., and F. Squintani (2010), "Competitive Experimentation with Private Information: The Survivor's Curse," Journal of Economic Theory, 145: 639-660.

[18] Piller, F.T. and D. Walcher (2006), "Toolkits for Idea Competition: A Novel Method to Integrate Users in New Product Development," REDD Management, 36: 307-318.

[19] Rieck, T. (2010), "Information Disclosure in Innovation Contests," Mimeo, University of Bonn.

[20] Seel C. and P. Strack (2013), "Gambling in Contests," Journal of Economic Theory, 148: 2033-2048.

[21] Seel C. and P. Strack (2016), "Continuous Time Contests with Private Information," Mathematics of Operations Research, 41: 1093-1107.

[22] Simon, L. and M. Stinchcombe (1989), "Extensive Form Games in Continuous Time, Part I: Pure Strategies," Econometrica, 57: 1171-1214.

[23] Taylor, C.R. (1995), "Digging for Golden Carrots: An Analysis of Research Tournaments," American Economic Review, 85: 872-890.

[24] Yildirim, H. (2005), "Contests with Multiple Rounds," Games and Economic Behavior, 51: 213-227.

[25] Zhang, J. and R. Wang (2009), "The Role of Information Revelation in Elimination Contests," Economic Journal, 119: 613-641. 